Published in final edited form as:

Nature. 2019 September ; 573(7774): 430-433. doi:10.1038/s41586-019-1546-Z.

\title{
Targeting Cardiac Fibrosis with Engineered T cells
}

Haig Aghajanian $1,2,3$, Toru Kimura ${ }^{4}$, Joel G. Rurik ${ }^{1,2,3}$, Aidan S. Hancock ${ }^{1,2,3}$, Michael S. Leibowitz ,5, Li Li1, 3 , John Scholler6, James Monslow7, Albert Lo7, Wei Han', Tao Wang', Kenneth Bedi', ${ }^{2,9}$ Michael P. Morley ${ }^{2,9}$, Ricardo A. Linares Saldana ${ }^{1,2,3}$, Nikhita A. Bolar ${ }^{1,2,3}$ Dirth ${ }^{11}$ CarI H. June 6 , Kenneth B. Margulies ${ }^{2}$, Rajan Jain ${ }^{2,3}$, Ellen Pure , Steven M. Albelda Jonathan A. Epstein 1,2,3,9

Department of Cell and Developmental Biology, Perelman School of Medicine at the University of Institute for Regenerative Medicine, Perelman School of Medicine at the University of Philadelphia, PA.

Department of Pediatrics, Perelman School of Medicine, University of Pennsylvania, Division of Oncology, The Children's Hospital of Philadelphia, Philadelphia, Pennsylvania

Center for Cellular Immunotherapies, Perelman School of Medicine at the University of

${ }^{7}$ Department of Biomedical Sciences, School of Veterinary Medicine at the University of Echocardiography Laboratory, Hospital of the University of Pennsylvania, Philadelphia, PA. Philadelphia, PA.

\footnotetext{
Users may view, print, copy, and download text and data-mine the content in such documents, for the purposes of academic research, subject always to the full Conditions of use:http://www.nature.com/authors/editorial_policies/license.html\#termsReprints and permissions information is available at www.nature.com/reprints.

*Address correspondence to: Jonathan Epstein, 602 South Tower, PCAM, 3400 Civic Center Blvd., Philadelphia, PA 19104, 215-898-8731, epsteinj@upenn.edu. Correspondence and requests for materials should be addressed to epsteinj@upenn.edu. Authors Contributions H.A. and J.A.E. conceived of the project and designed experiments. H.A., T.K., J.G.R., A.S.H., M.S.L., L.L., J.M., A.L., W.H., T.W., K.B, R.A.L.S., N.A.B, K.M., C-A.A., and C.L.S. performed experiments and interpreted data. M.P.M. performed bioinformatic analysis and interpretation. J.S., R.J., D.W. C.H.J., K.B.M., E.P., and S.M.A. contributed reagents, analysis, and interpretation. H.A. and J.A.E. wrote the manuscript. J.A.E. supervised all aspects of the research. Author Information C.H.J., E.P, and S.M.A. are inventors (University of Pennsylvania, Wistar Institute) on a patent for a FAP CAR (US Utility Patent 9,365,641 issued June 14, 2016, WIPO Patent Application PCT/US2013/062717). H.A. and J.A.E. are inventors (University of Pennsylvania) on a patent for the use of CAR T therapy in heart disease (US Provisional Patent Application 62/563,323 filed 9/26/2017, WIPO Patent Application PCT/US2018/052605). C.H.J. reports grants from Novartis and Tmunity Therapeutics.

C.H.J. is a scientific founder and has equity in Tmunity Therapeutics, a biotech dedicated to developing engineered T cells for therapy of cancer, infections and autoimmunity. C.H.J. is on the scientific advisory boards of Immune Design, Viracta Therapeutics, Carisma Therapeutics, and Cabaletta Bio.

Supplementary Information is linked to the online version of the paper.
} 
${ }^{10}$ Department of Pathobiology, School of Veterinary Medicine at the University of Pennsylvania, Philadelphia, PA.

${ }^{11}$ Model Systems for Infection and Immunity, Helmholtz Centre for Infection Research, Braunschweig, Germany.

\section{Summary}

Fibrosis is observed in nearly every form of myocardial disease ${ }^{1}$. Upon injury, cardiac fibroblasts $(\mathrm{CF})$ in the heart begin to remodel the myocardium via extracellular matrix deposition, resulting in increased tissue stiffness and reduced compliance. Excessive cardiac fibrosis is an important factor in the progression of various forms of cardiac disease and heart failure ${ }^{2}$. However, clinical interventions and therapies targeting fibrosis remain limited ${ }^{3}$. In this study, we demonstrate the efficacy of redirected T-cell immunotherapy to specifically target pathologic cardiac fibrosis. We find that cardiac fibroblasts expressing a xenogeneic antigen can be effectively targeted and ablated by adoptive transfer of antigen-specific $\mathrm{CD} 8^{+} \mathrm{T}$ cells. Through expression analysis of cardiac fibroblast gene signatures from healthy versus diseased human hearts, we identified an endogenous CF target; fibroblast activation protein (FAP). Adoptive transfer of T cells expressing a chimeric antigen receptor (CAR) against FAP, results in a significant reduction in cardiac fibrosis and restoration of function after injury in mice. These results provide the proof-of-principle basis for a novel immunotherapeutic avenue for the treatment of cardiac disease.

Although quiescent fibroblasts are an important component of the normal structure of the myocardium, activated, pathological fibroblasts induced by injury or disease negatively impact compliance and stiffness, and signal to cardiac myocytes to further negatively impact function ${ }^{4}$. Nevertheless, no therapies are known to directly target excessive fibrosis ${ }^{5}$, and very few interventions have been shown to improve cardiac function and clinical outcomes in patients with impaired cardiac compliance and relaxation causing heart failure including those with HFpEF (heart failure with preserved ejection fraction) despite the enormous burden of disease across the population ${ }^{6}$. However, genetic ablation of cardiac fibroblasts after hypertensive or ischemic injury has been shown to reduce fibrosis and improve function in mice ${ }^{7,8}$, providing further support for targeting these cells directly.

Recently, breakthroughs in cancer therapy have been achieved by redirecting cytotoxic $\mathrm{T}$ cells to recognize specific antigens on cancer cells using either a modified T-cell receptor $(\mathrm{TCR})^{9}$ or a chimeric antigen receptor (CAR $)^{10}$. The modified T cells are capable of effectively eliminating the cancer cells, and CAR-T therapy has been approved by the FDA $^{11}$ for use in some forms of human leukemia and lymphoma ${ }^{12}$. We and others ${ }^{13,14}$ reasoned that engineered $\mathrm{T}$ cells may possibly be used in targeting non-cancer cells, and we sought to determine if activated cardiac fibroblasts could be effectively targeted by engineered $\mathrm{T}$ cells in a mouse model of heart disease.

In a first series of experiments, we generated mice in which a xenogeneic antigen (ovalbumin peptide, OVA) ${ }^{15,16}$, not normally found in mice, could be conditionally presented on the surface of activated cardiac fibroblasts. To induce expression of OVA, we utilized a tamoxifen (Tam) inducible Cre recombinase targeted to the Periostin locus $\left(\right.$ Postn $\left.{ }^{M C M}\right)$. Postn has been shown to be expressed by activated cardiac fibroblasts induced 
by injury, but not by quiescent cardiac fibroblasts ${ }^{8}$. In order to induce injury and fibrosis, OVA;Postn ${ }^{M C M}$ mice were continuously infused with angiotensin II and phenylephrine (AngII/PE) for 28 days via osmotic minipump. Tamoxifen was administered at regular intervals by intraperitoneal injection to ensure near constant induction of Cre recombinase and expression of ovalbumin (Fig. 1a). AngII/PE administration has been widely demonstrated to induce dramatic cardiac fibrosis and dysfunction in mice by increasing afterload, chronotropy, and direct effects on cardiac myocytes and fibroblasts ${ }^{7,8}$. Consistent with previous reports demonstrating a peak of cardiac fibrosis within 1 week of this type of injury, we observed significant and widespread fibrosis throughout the myocardium of OVA;Postn ${ }^{M C M}$ mice one week following exposure to AngII/PE (Extended Data Fig. 1a). To selectively target OVA-expressing cardiac fibroblasts and their derivatives, we performed adoptive transfer of $\mathrm{CD} 8^{+}$cells that express a cognate T-cell receptor against the OVA peptide $\left(\mathrm{CD} 8^{+} \text {OT-I T cells }\right)^{17,18}$ in a cohort of mice at this time point (1 week after AngII/PE initiation) once pathological fibrosis was established. Control animals included $O V A ; P{ } t_{n}{ }^{M C M}$ mice that received AngII/PE and tamoxifen, as well as $O V A+$ mice lacking the Postr ${ }^{M C M}$ allele that received AngII/PE, tamoxifen, and CD8+ OT-I cells ( $\mathrm{n} \geq 6$ mice in each cohort). At week 4, we observed continued widespread cardiac fibrosis in the control animals. However, in marked contrast, there was significantly less cardiac fibrosis in the OVA;Postn ${ }^{M C M}+$ Tam mice treated with OT-I T cells (Fig. 1b, c). In addition to the reduced fibrosis, an index correlating with cardiac hypertrophy, heart-weight to body-weight ratio, was partially rescued after OT-I T cell adoptive transfer in $O V A ; P o s t n^{M C M}$ mice (Extended Data Fig. 1b, c).

Having demonstrated that immunologically targeting and depleting activated cardiac fibroblasts is possible, we sought to identify endogenous proteins/antigens expressed by activated cardiac fibroblasts, but not by quiescent fibroblasts or other critical cell types, that could be specifically targeted by engineering (or genetically modifying) $\mathrm{T}$ cells to recognize such an antigen. We analyzed gene expression data from an RNAseq database compiled from samples of 238 human heart transplant donor and recipient left ventricular (LV) tissue. We found several fibroblast-specific genes ${ }^{19,20}$ that are upregulated in myocardium from patients with either hypertrophic cardiomyopathy (HCM) or dilated cardiomyopathy (DCM) when compared to control, non-failing donor hearts (Fig. 2a, Extended Data Fig. 2a). Fibroblast activation protein (FAP) showed the greatest fold change and upregulation when comparing either DCM or HCM samples to controls. FAP is a cell surface glycoprotein ${ }^{21}$ that has been shown to be expressed during embryonic development at sites of active tissue remodeling such as during wound repair, in tissue fibrosis, and in various tumors, but is expressed at only low levels if at all in most normal adult tissues of mouse or human ${ }^{22,23}$. FAP expression has been previously observed in human hearts following acute myocardial infarction injury ${ }^{24}$. We extended this finding by showing that FAP is robustly expressed by cardiac fibroblasts (and not by myocytes) in the LV tissue from failing DCM and HCM human hearts (Fig. 2b, Extended Data Fig. 2b). Normal human hearts demonstrate minimal FAP expression (Fig. 2b). Thus, FAP is a potential candidate for targeting of pathological cardiac fibroblasts.

To test the feasibility of FAP as an endogenous CF target for immunotherapy, we returned to the AngII/PE model of hypertensive cardiac injury and fibrosis in mice. As we observed in 
our previous experiments, there is robust cardiac fibrosis as early as 1 week after AngII/PE infusion in C57B1/6 mice, and we confirmed that Fap is expressed by the activated cardiac fibroblasts in this model of injury (Fig 2c, Extended Data Fig. 3a). Immunohistochemistry showed that Fap is not detectable in control hearts, with little to no expression elsewhere in the body, but is apparent on activated fibroblasts after 1 and 2 weeks of AngII/PE exposure, as well as additional models of cardiac injury including myocardial infarction, transverse aortic constriction, and muscular dystrophy (Fig 2c, Extended Data Fig. 3b-d). To target and deplete Fap-expressing cardiac fibroblasts in the AngII/PE model, we adoptively transferred FAP CAR T cells at these 1 and 2 week timepoints after AngII/PE initiation (Fig. 3a). We administered a second injection of FAP CAR T cells in this set of experiments due to the short half-life of mouse FAP CAR T cells that we have previously observed in tumor models ${ }^{25}$. We detected FAP CAR T cells infiltrating the myocardium and co-localizing with Fap-expressing CFs within 1 day of adoptive transfer (Extended Data Fig. 4a). By 4 weeks, there was a significant reduction of cardiac fibrosis in injured mice that had been treated with the FAP CAR T cells when compared to controls (Fig. 3b, c), and remained limited at 8 weeks $(1.92 \% \pm 0.25, \mathrm{n}=7)$. Continued and widespread cardiac fibrosis was evident in each of the control mice that were exposed to AngII/PE, while fibrosis was reduced in all 7 of 7 (and nearly eliminated in 5 of 7) AngII/PE-exposed mice that were treated with FAP CAR T cells (Extended Data Fig. 4b-d). Along with the reduction in fibrosis, we observe a partial rescue of both systolic and diastolic cardiac function in injured mice treated with FAP CAR T cells when compared to controls (Fig. 3d, e, Extended Data Fig. 5). Interestingly, we noted persistence of perivascular fibrosis after treatment, consistent with the lack of Fap expression in perivascular fibroblasts (Extended Data Fig. 6a, b) induced by AngII/PE.

CAR T cells against FAP have been used safely and effectively by multiple groups in cancer treatment studies involving mice ${ }^{25-28}$ and have been administered to humans as part of a clinical trial for the treatment of mesothelioma ${ }^{29}$. Nevertheless, we performed extensive analysis of potential toxicities in our model system. We were not able to detect any histological effects of FAP CAR T cell therapy in numerous non-cardiac organs or tissues (Extended Data Fig. 6c), consistent with previous studies from our group in which we targeted tumor stromal cells using FAP CAR T cells without untoward effects ${ }^{25,27}$. Serum cytokine levels, including interleukin 2 and 6, were increased upon AngII/PE administration, but adoptive transfer of FAP CAR T cells did not further increase cytokine levels which we monitored for 12 weeks after treatment (Extended Data Fig. 7). In order to determine if FAP CAR T cell treatment might be itself cardiotoxic, we utilized a commercially available cardiotoxicity array to interrogate 84 genes associated with myocardial toxicity. At 4 weeks, gene expression differences between AngII/PE and AngII/PE + FAP CAR T cell-treated animals were noted although the changes were only partially consistent with cardiotoxicity (Extended Data Fig. 8a). These differences resolved by 8 weeks (Extended Data Fig. 8b). Further, we assessed the expression levels of 1659 inflammatory and immune genes and found only 22 genes differentially expressed between AngII/PE and AngII/PE +FAP CAR T cell conditions at 4 weeks suggesting only very mild inflammation (Extended Data Fig 8c). Immunohistologic analysis for immune cell infiltration revealed no significant differences in $\mathrm{CD} 3+, \mathrm{CD} 4+, \mathrm{CD} 8+$, or MPO+ cells, a reduction in CD19+ cells, and an increase in $\mathrm{F} 4 / 80+$ macrophages in AngII/PE +FAP CAR T compared to AngII/PE-treated samples at 4 weeks 
(Extended Data Fig. 8d). All FAP CAR T cell treated animals survived to 12 weeks with no evidence of toxicity (Extended Data Fig. 9a-c).

In separate experiments, we examined the effect of FAP CAR $\mathrm{T}$ therapy on wound healing since FAP is expressed by some, but not all, fibroblasts in the $\operatorname{skin}^{30}$. No delays or defects in wound healing were observed in FAP CAR T versus control-treated animals (Extended Data Fig. 9d-f), consistent with the presence of multiple fibroblast populations in various tissues, some of which do not express FAP ${ }^{31}$. Although rare FAP-expressing cells have been reported in the pancreas, no change in serum amylase levels were observed in response to FAP CAR T treatment (Extended Data Fig. 9g).

These results provide proof-of-concept for the possibility of targeting cardiac fibrosis in mice with engineered $\mathrm{T}$ cells ${ }^{32}$ and immunotherapy. However, before this approach can be translated to humans, much work remains to determine if FAP is the optimal target and that safety risks are minimized (Supplementary Table 1). Additionally, specific CAR constructs against the same antigen can have vastly different affinities, efficacies, and safety profiles $^{25,27,33}$. Continued investigation to identify unique antigens expressed by activated cardiac fibroblasts may identify alternative antigens, or combinations of antigens, that may be effective as targets for immunotherapy in humans with heart disease. As with any T cell therapy, off target effects can range from mild to serious ${ }^{34,35}$. It may be necessary to engineer modified T cells with a "kill-switch" to limit time of survival so as to minimize any off-target side-effects. These approaches are under development ${ }^{36}$. Enormous progress in the treatment of certain cancers has been achieved through the use of engineered T cells. Here, we suggest that the "immunorevolution" may extend beyond oncology to impact one of the most common forms of human morbidity and mortality: heart disease.

\section{Methods}

\section{Mice}

Periostin ${ }^{M C M}$ mice were obtained from the laboratory of Jeffery Molkentin ${ }^{37}$ (Cincinnati Children's). RosaOVA mice provide Cre-dependent expression of an intracellular ovalbumin epitope that is presented via MHC $\mathrm{I}^{38,39,40} . M d x / m T R^{K O}$ mice were obtained from the laboratory of Foteini Mourkioti ${ }^{41}$. C57BL/6 mice were obtained from Charles River Laboratories (Wilmington, MA). OT-I mice were obtained from The Jackson Laboratory (Bar Harbor, ME). Sample sizes were determined by power calculations based on effect sizes previously reported in the literature. This study complied with all relevant ethical regulations and all animal protocols were approved by the University of Pennsylvania Institutional Animal Care and Use Committee (IACUC).

\section{Reagents and antibodies}

Osmotic mini-pumps (Alzet, model 2004) were used for drug delivery. Angiotensin II (A9525-50MG), phenylephrine hydrochloride (P6126-10G), tamoxifen (T5648-1G), and corn oil (C8267) were obtained from Millipore Sigma. Picro Sirius Red Stain Kit (ab150681), Anti-Fibroblast activation protein, alpha antibody (ab207178), anti-GFP antibody (ab6673), and anti-CD4 antibody (183685) were obtained from Abcam. Anti- 
Vimentin antibody (\#9854) anti-F4/90 antibody (\#70076), anti-CD19 antibody (\#90176), and anti-CD8 antibody (\#98941) were obtained from Cell Signaling Technology. AntiTroponin T antibody (MS-295) was obtained from ThermoFisher Scientific. Anti-CD3 antibody (MCA1477T) was obtained from Bio-Rad Laboratories. Anti-Myeloperoxidase (MPO) antibody (A0398) was obtained from Dako/Agilent. Sterile saline (0.9\% sodium chloride, 00409488850) was obtained from Hospira (Lake Forest, IL).

\section{Cardiac Injury}

All mice used in these experiments were adult males between 10 and 14 weeks of age. WT mice were randomized to different conditions. Angiotensin II $(1.5 \mu \mathrm{g} / \mathrm{g} / \mathrm{day})$ and phenylephrine $(50 \mu \mathrm{g} / \mathrm{g} / \mathrm{day})$ or saline ( $0.9 \%$ sodium chloride) were continuously administered through osmotic mini-pump (Alzet, 2004) for 28 days. Periostin ${ }^{M C M}$ mice were induced for Cre expression by intraperitoneal injections of $100 \mu$ of tamoxifen in corn oil $(20 \mathrm{mg} / \mathrm{ml})$. Injections were administered on alternating days for 1 week, and then weekly afterward until sacrificed. Adoptive transfer of $\mathrm{T}$ cells was administered through tail vein injection. Echocardiography performed under isoflurane anesthesia using a FUJIFILM VisualSonics system with an MS400 probe and Vevo 2100 software, and analyzed blinded to treatment group.

For myocardial infarction, animals are anesthetized and a thoracotomy is performed by separating the fourth or fifth intercostal space to expose the heart. The pericardium is opened and an 8-0 nylon ligature, entering the heart on the left margin of the pulmonary cone and exiting near the insertion of the left auricular appendage is tied on left anterior descending coronary artery (LAD) to induce permanent myocardial infarction (MI).

For transverse aortic constriction (TAC), animals are anesthetized and antiseptic agents (Betadine and 70\% ethenol) are applied at the surgical area. The chest cavity is opened by an incision of the left second intercostal space. The transverse aorta is dissected from the surrounding tissues and a 7-0 silk suture is passed underneath of the arch portion of the aorta and ligated for creation of stenosis of the aorta with about 50-70\% lumen constriction.

\section{Adoptive T cell transfer}

Mouse $\mathrm{T}$ cells expressing a CAR construct specific for mouse FAP (containing the $\mathrm{scFv}$ fragment from the specific mouse FAP antibody (clone 73.3) (2) $^{42}$ coupled to the human CD3 $\zeta$ and CD28 cytoplasmic domains) along with a small peptide that conferred resistance to adenosine and prostaglandin E2 mediated suppression ${ }^{43}$ or GFP were used. Infective particles were generated from the supernatants of "Phoenix" packaging cells (Allele; Phoenix Eco Cells Line \#ABP-RVC-10002; purchased from and authenticated by ATCC, CRL-3214; routinely tested for mycoplasma - negative) transfected with retroviral vector plasmid and helper plasmids using Lipofectamine 2000 (Invitrogen), as previously described $^{42}$. T cells were cultured in RPMI 1640 supplemented with $10 \%$ FBS, $100 \mathrm{U} / \mathrm{mL}$ penicillin, $100 \mu \mathrm{g} / \mathrm{mL}$ streptomycin sulfate, $1 \mathrm{mM}$ Pyruvate, and $50 \mu \mathrm{M}$ betamercaptoethanol. Primary murine $\mathrm{T}$ cells were isolated as suggested by the manufacturer (Miltenyi Biotec) from the spleens of mice and incubated in 12 -well plates $\left[2 \times 10^{6}\right.$ cells/well in $2 \mathrm{ml} \mathrm{T}$-cell media with $50 \mathrm{U} / \mathrm{mL}$ mouse interleukin (IL)-2 and Dynabeads Mouse T- 
Activator CD3/CD28 (Gibco, \#11453D) in a 1:1 ratio]. After 48 hours, cells were plated in a 24-well plate coated with retrovirus and $5 \mu \mathrm{g} / \mathrm{cm}^{2}$ of Retronectin (Takara, \#T100B). Prior to plating T cells, a Retronectin-coated 24-well plate was centrifuged without braking at room temperature for 1 hour at $1000 \mathrm{~g}$ with $1 \mathrm{ml} /$ well crude viral supernatant. After overnight incubation, cells were expanded with $50 \mathrm{U} / \mathrm{mL}$ of IL-2 for 48 hours. The transduction efficiency with muFAP CAR-RIAD (FAP CAR T cells), FAP-GFP-CAR or MigR1-GFP (control $\mathrm{T}$ cells) was assessed after transduction. $10^{7} \mathrm{CAR} \mathrm{T}$ cells were injected per mouse.

For the OVA experiments, cells were obtained from C57BL/6 OT-I mouse spleens and purified using the mouse CD8a+ T Cell Isolation Kit (Miltenyi Biotec, 130-104-075). Purity was assessed by flow cytometry. $5 \times 10^{6} \mathrm{CD} 8+\mathrm{T}$ cells were adoptively transferred to mice 1 week after injury.

\section{Histology and Immunohistochemistry}

Mouse and human tissue samples fixed overnight in 2-4\% paraformaldehyde and dehydrated through an ethanol series. All samples were paraffin-embedded and sectioned. Hematoxylin and eosin (H\&E), and Masson's trichrome staining were completed using a standard protocol. Pricro Sirus Red staining was completed using a kit (Abcam, 150681) as per manufacturer's instructions. Briefly, sections were deparaffinized, incubated with Pircro Sirius Red for 1 hour, washed in acetic acid solution, washed in absolute alcohol, cleared with xylene, and mounted. Slides were digitally scanned at 20x and analyzed via color deconvolution using ImageScope (Aperio) software. At least eight distinct sections were quantified for percent fibrosis for each mouse in each condition.

\section{Human Cardiac Expression data}

Failing and nonfailing human heart tissues were obtained from heart transplant recipients and from brain-dead organ donors. In situ cardioplegia was employed for cardioprotection in all human heart procurements, as previously described ${ }^{44,45}$. In vivo echocardiography was performed prior to tissue harvest for contemporaneous assessment of in vivo structure and function. Procurement of human myocardial tissue was performed with consent from all subjects and under protocols and ethical regulations approved by Institutional Review Boards at the University of Pennsylvania and the Gift-of-Life Donor Program (Pennsylvania, USA). Tissue specimens were frozen in liquid nitrogen and stored at $-80^{\circ} \mathrm{C}$ until used.

Total RNA was extracted from human cardiac tissue samples using the miRNeasy Kit (Qiagen) including DNAse treatment. For RNA-seq, library prep was conducted using Illumina truSeq stranded mRNA kit followed by the Nugen Ovation amplification kit. Resultant fastq files were assessed for quality control using the FastQC program. Fastq files were aligned against the human reference genome (hGRC37/Hg19) using the STAR aligner ${ }^{46}$. Duplicate reads were flagged using the MarkDuplicates program from Picard tools. Per gene read counts for Ensembl (v75) gene annotations were computed using the R package with duplicate reads removed. Gene counts represented as counts per million (CPM) were first nominalized using TMM method in the edgeR R package and genes with $25 \%$ of samples with a CPM $<1$ were removed and deemed low expressed. The data was 
transformed using the VOOM function from the limma R package ${ }^{47}$. Differential gene expression was performed using a linear model with the limma package.

\section{Cardiotoxicity Assay}

Total RNA was isolated from formalin fixed, paraffin-embedded cardiac sections with RNeasy FFPE kit (Qiagen 73504, 19093). Samples were blinded to condition. cDNA was then prepared with the RT ${ }^{2}$ PreAMP cDNA synthesis kit (Qiagen 330451) and pre-amplified with assay-specific RT ${ }^{2}$ PreAMP primer mix (Qiagen 330241 PBM-095Z) according to the manufacturer's recommendations. Pre-amplified cDNA was then assayed using the mouse cardiotoxicity $\mathrm{RT}^{2}$ profiler PCR array (Qiagen 330231 PAMM095Z) with $\mathrm{RT}^{2}$ SYBR Green ROX qPCR mastermix (Qiagen 330523) on a real-time PCR StepOnePlus system (Applied Biosystems). The threshold was set consistently across the entire experiment. Measured $\mathrm{Ct}$ values were normalized to Gapdh, compared using Welch's t-test, and graphed in R using the tidyverse suite of packages.

\section{Cytokine Assay}

Blood was collected by cardiac puncture or via IVC during dissection at 10 days, 2 weeks (14 days), 4 weeks (28 days), and 12 weeks (84-88 days), allowed to clot, and spun down to isolate serum. Blinded serum samples were assayed by multiplex technology (MAGPIX, Luminex Corp, Austin TX) by overnight incubation using a mouse cytokine 25plex (mcytomag-70k-PMX, EMD Millipore).

\section{Immune and Inflammation assay}

Immune and inflammation-related gene expression analyses were done with the ImmunoOncology assay (HTG Molecular Diagnostics, Tucson, AZ, USA). Briefly, samples were obtained from formalin-fixed paraffin-embedded sections of hearts from mice that had been treated with AngII/PE +FAP CAR T cells or AngII/PE for 4 weeks ( $\mathrm{n}=3$ per condition, blinded before processing). The estimated mean value of each probe for each group was normalized using DESeq2 median normalization ${ }^{48}$. The p-value for each probe was adjusted using the Benjamini and Hochberg method for controlling the false discovery rate ${ }^{49}$. The estimated mean value of each probe for each group after normalization is displayed as a Volcano plot of the $\log [10]$-transformed adjusted p-value against the log-fold change for each probe in the assay.

\section{Wound Healing Assay}

8-9 week-old C57BL/6 mice were anesthetized using isoflurane and two equidistant 4-mm full-thickness excisional wounds were generated using circular biopsy punches in the dorsal skin approximately $5 \mathrm{~mm}$ below the shoulder blades. Mice were randomly assigned into two groups ( $\mathrm{n}=6$ animals per group) and received $1 \times 10^{7}$ control or FAP-CAR-transduced mouse $\mathrm{T}$ cells intravenously immediately after wounding and three days post-wounding. Body weight measurements were taken every other day along and with imaging of the wounds using the IVIS Lumina III Bioluminescence and Fluorescence Imaging System (PerkinElmer, Waltham MA). Mice were euthanized eight days post-wounding. Serum was collected to measure amylase levels as a read-out for pancreatic function. Skin samples were 
fixed in prefer fixative (Anatech Ltd, Battle Creek, MI) stained with and H\&E. Digital images were captured on a Nikon TiE inverted microscope (Nikon Instruments Inc., Melville, NY). Wound closure was quantified using ImageJ software.

Statistics

Differences between two groups were compared using Student's or Welch's t-test.

Differences between multiple groups were compared with one-way analysis of variance (ANOVA). Significant ANOVA results were further analyzed by Tukey's multiple comparisons test. $* P<0.05, * * P<0.01, * * * P<0.001$, ns $=$ not significant.

\section{Data Availability}

All data are available from the corresponding author upon request.

\section{Extended Data}



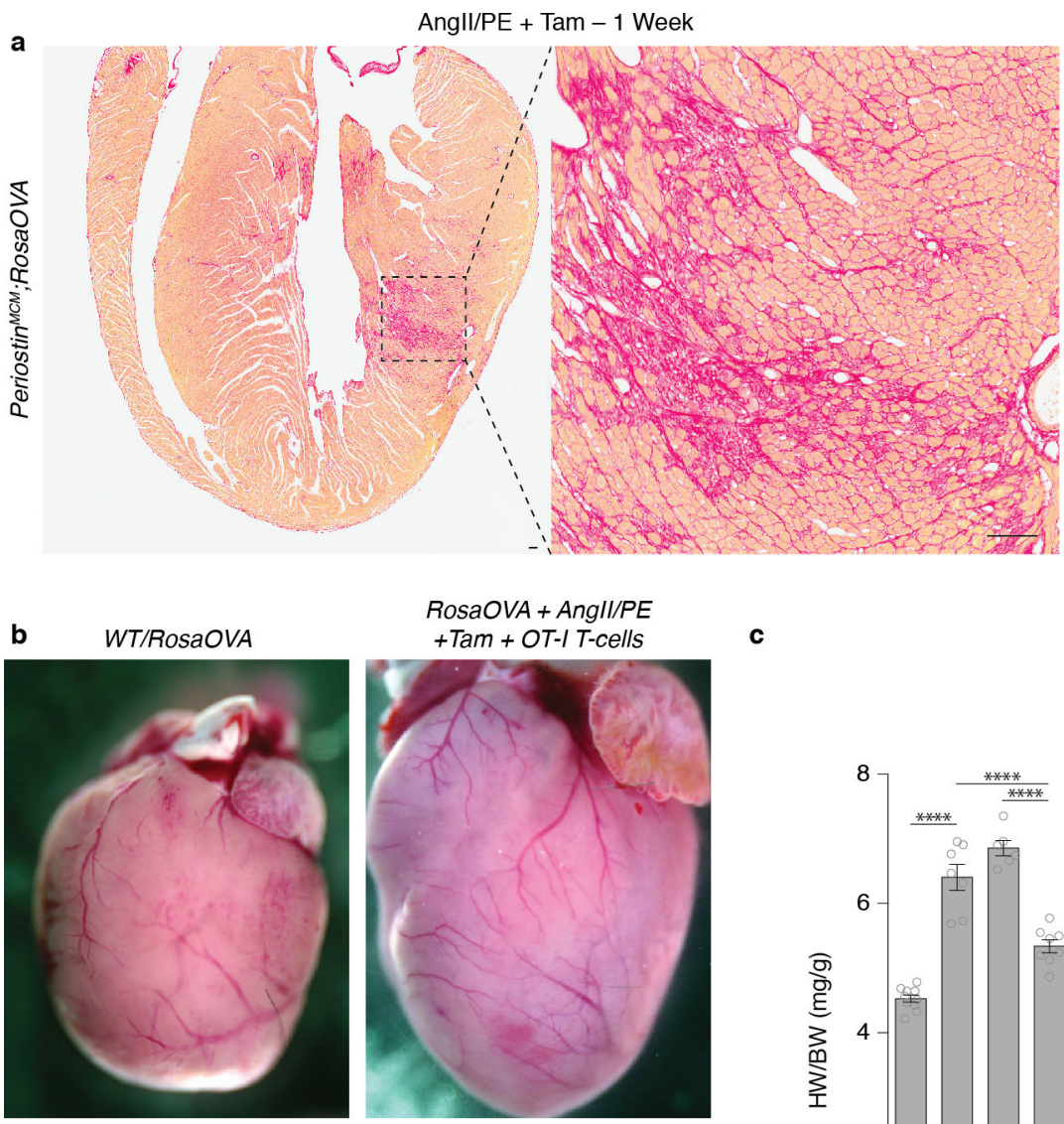

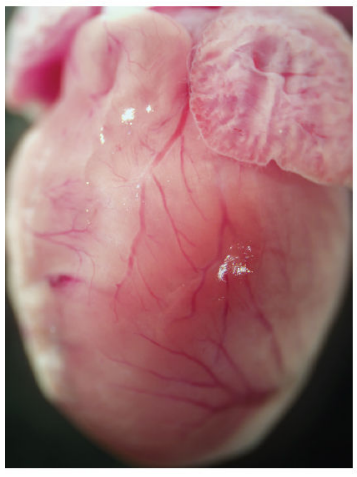

Pstr ${ }^{M C M} ;$ RosaOVA + AngIl/PE + Tam

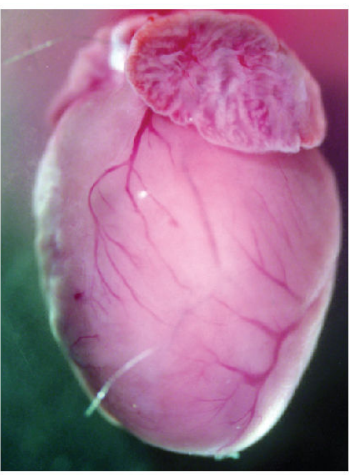

Pstn ${ }^{M C M} ;$ RosaOVA + Angll/PE + Tam + OT-I T-cells $\mathbf{c}$

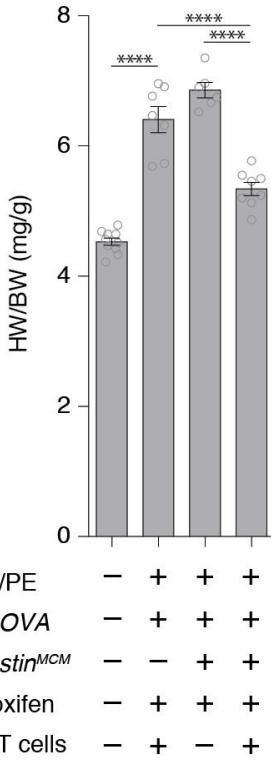

Extended Data Fig. 1.

Cardiac fibrosis and hypertrophy.

(a) Pircro-Sirius Red staining for cardiac fibrosis (red) in a heart coronal section from a Periostin $^{\text {MCM; }}$;osaOVA mouse treated with AngII/PE and tamoxifen for 1 week. High powered field of left ventricular free-wall (right). Representative image of 3 biologically independent animals with similar results. (b) Control and experimental hearts were measured (weight, mg) and images captured. (c) Quantification of heart weight to body weight $(\mathrm{HW} / \mathrm{BW})$ ratio of indicated genotypes and conditions (mean $\pm \mathrm{SEM})$. $* * * * P<$ 0.0001 (one-way ANOVA between groups $P<0.0001$; post-hoc multiple comparisons, 
Tukey's test, $\mathrm{n}=10,7,6,8$ biologically independent animals, respectively). Scale bars $=$ $100 \mu \mathrm{m}$. 


\begin{tabular}{lcccc} 
a & \multicolumn{2}{c}{ HCM } & \multicolumn{2}{c}{ DCM } \\
\cline { 2 - 5 } Gene & Fold Change & $P$ Value & Fold Change & $P$ Value \\
\hline Symbol & & & & \\
\hline FAP & 3.92 & $6.97 \mathrm{E}-28$ & 3.11 & $3.12 \mathrm{E}-36$ \\
THY1 & 3.92 & $6.40 \mathrm{E}-20$ & 3.12 & $5.07 \mathrm{E}-27$ \\
POSTN & 2.64 & $1.40 \mathrm{E}-11$ & 2.02 & $3.73 \mathrm{E}-13$ \\
COL1A1 & 2.22 & $1.83 \mathrm{E}-15$ & 2.71 & $1.04 \mathrm{E}-41$ \\
WT1 & 1.74 & $6.29 \mathrm{E}-12$ & 1.57 & $4.17 \mathrm{E}-16$ \\
COL3A1 & 1.53 & $1.28 \mathrm{E}-05$ & 1.77 & $8.72 \mathrm{E}-18$ \\
TCF21 & 1.39 & $1.01 \mathrm{E}-05$ & 1.59 & $5.26 \mathrm{E}-19$ \\
VIM & 1.26 & $1.43 \mathrm{E}-12$ & 1.20 & $1.60 \mathrm{E}-16$ \\
ACTA2 & 1.26 & $2.64 \mathrm{E}-03$ & 1.22 & $7.16 \mathrm{E}-05$ \\
DDR2 & 1.05 & 0.21 & 1.02 & 0.47 \\
S100A4 & -1.00 & 0.96 & -1.03 & 0.33 \\
FN1 & -1.01 & 0.90 & 1.11 & 0.06 \\
P4HA1 & -1.18 & $4.11 \mathrm{E}-04$ & -1.11 & $1.10 \mathrm{E}-03$ \\
PDGFRA & -1.25 & $1.40 \mathrm{E}-05$ & -1.17 & $9.98 \mathrm{E}-06$ \\
\hline
\end{tabular}
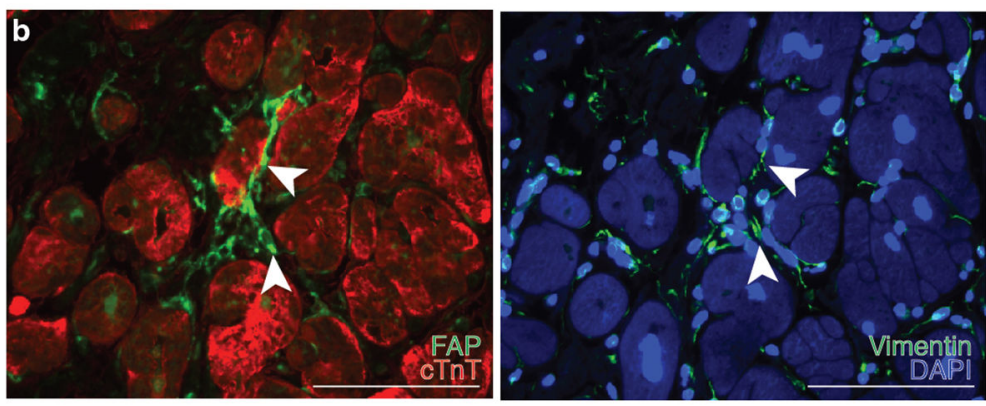

\section{Extended Data Fig. 2.}

Markers of activated cardiac fibroblasts in human disease.

(a) Fold change and $P$ values of cardiac fibroblast-specific gene expression from patients with hypertrophic cardiomyopathy (HCM) and dilated cardiomyopathy (DCM) as compared with non-failing hearts. ( $\mathrm{n}=122$ (non-failing), 27 (HCM), 89 (DCM)) Differential gene expression was performed using a linear model. (b) Immunohistochemistry co-staining cardiac Troponin (red) and FAP (green; left) and Vimentin (green; right) in adjacent sections from the left ventricle of a human patient with cardiomyopathy. FAP and Vimentin are seen on the same fibroblasts (arrowheads). Representative image of 2 independent experiments with similar results. HCM= Hypertrophic cardiomyopathy, DCM = Dilated cardiomyopathy. Scale bars $=100 \mu \mathrm{m}$. 


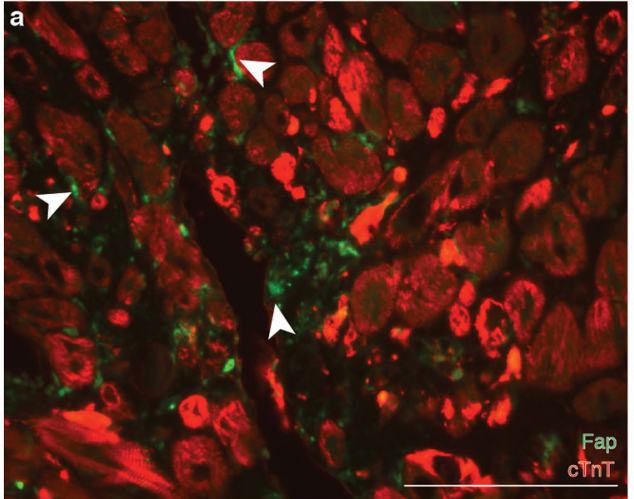

b

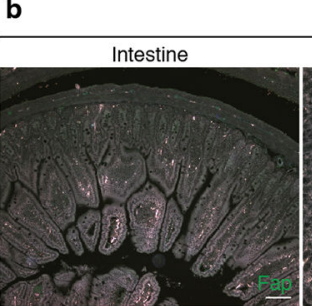

Lung

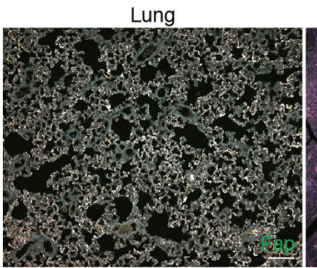

Spleen

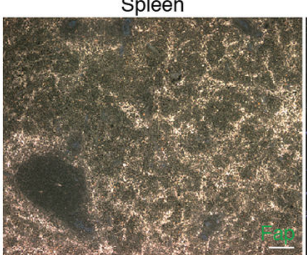

MI

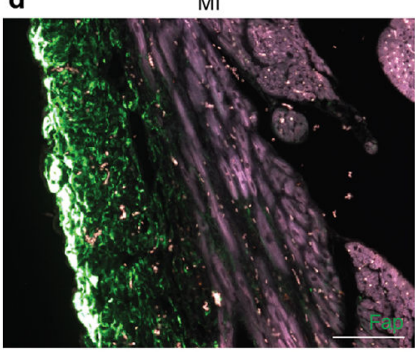

Angll/PE - 1 Week

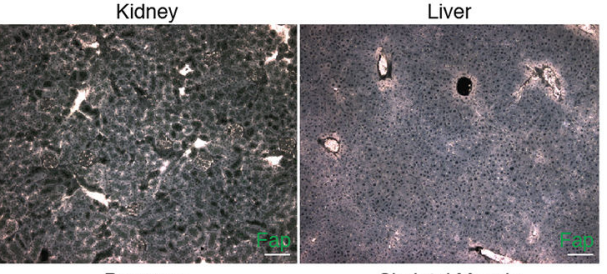

Skeletal Muscle

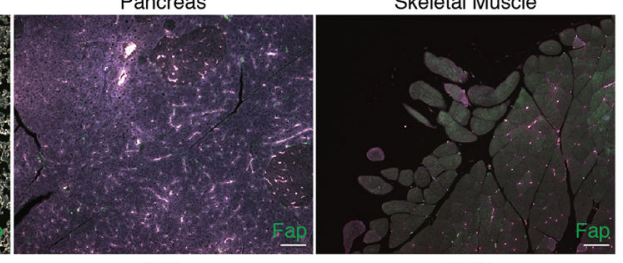

Skin

Testis

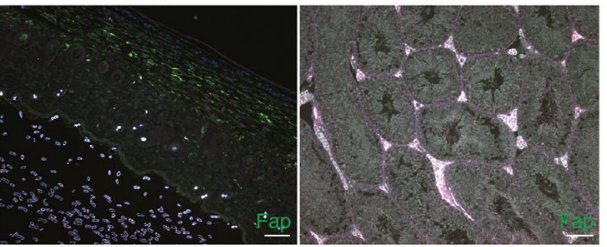

TAC

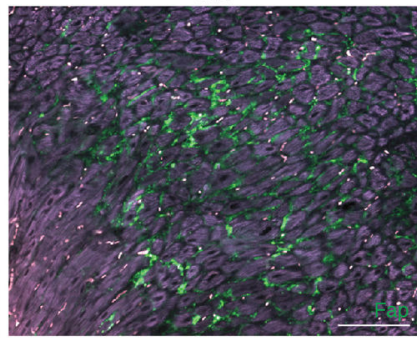

Angll/PE - 2 weeks
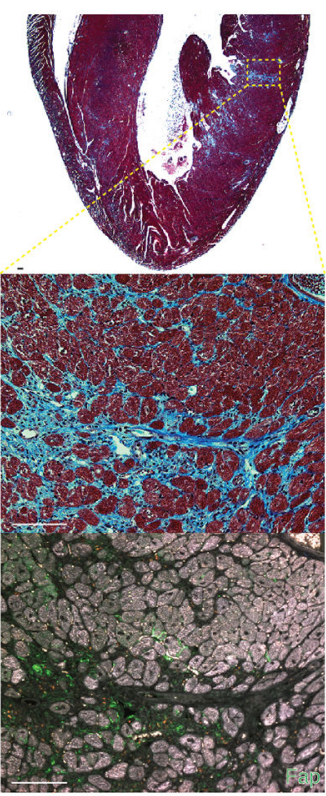

DMD

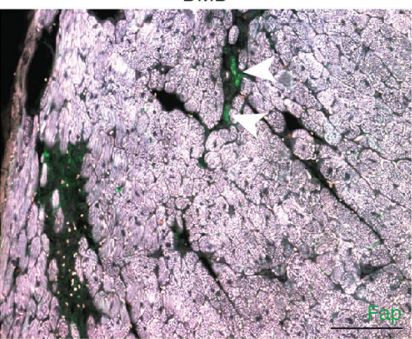

Extended Data Fig. 3.

Fap is expression in mouse cardiac fibroblasts after injury.

(a) Immunohistochemistry co-staining cardiac Troponin (red) and FAP (green; left) and Vimentin (green; right) in adjacent sections from the left ventricle of a mouse treated with AngII/PE for 2 weeks. Fap and Vimentin are seen on the same fibroblasts (arrowheads). Representative images from 2 independent experiments with similar results $(\mathrm{n}=7$ biologically independent animals). (b) Immunohistochemistry for Fap (green) in various organs/tissues after 1 week of AngII/PE treatment. Representative image of 3 biologically 
independent animals with similar results. (c) Masson's trichrome stain for fibrosis (blue; top, center) and immunohistochemistry for Fap (green; bottom) in WT coronal heart sections 2 week after continuous AngII/PE treatment. Staining and immunohistochemistry performed in adjacent sections. Bottom insets: higher magnification of left ventricular free-wall.

Representative image from 2 independent experiments with similar results ( $\mathrm{n}=7$ biologically independent animals). (d) Immunohistochemistry for Fap (green) in mouse models of cardiac injury. MI: myocardial infarction, TAC: transverse aortic constriction, DMD: Duchenne's muscular dystrophy $\left(m d x / m T R^{K O} \mathrm{G} 2\right.$ mice $)$. Scale bars $=100 \mu \mathrm{m}$. 
a ${ }_{\text {Control + FAP-GFP CART cells }}$

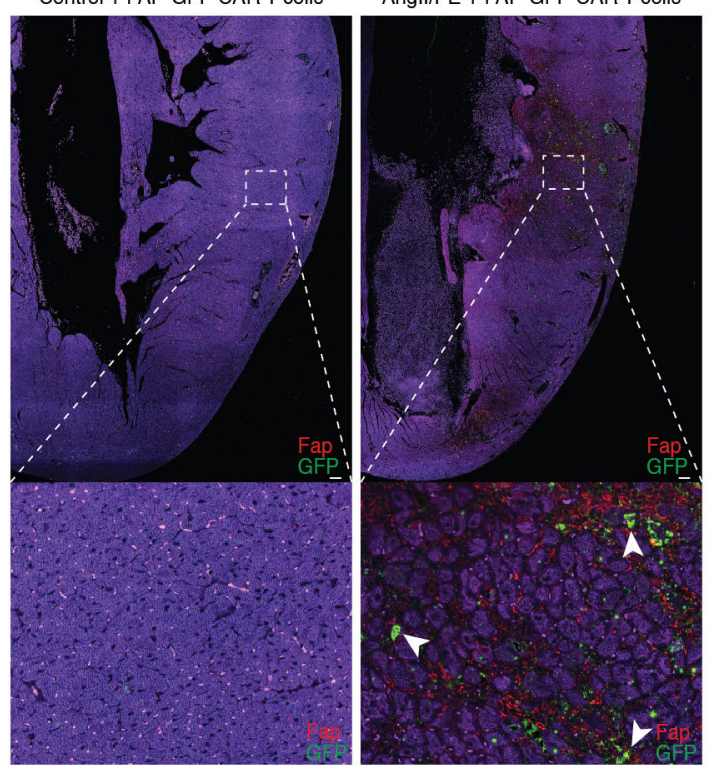

Angll/PE

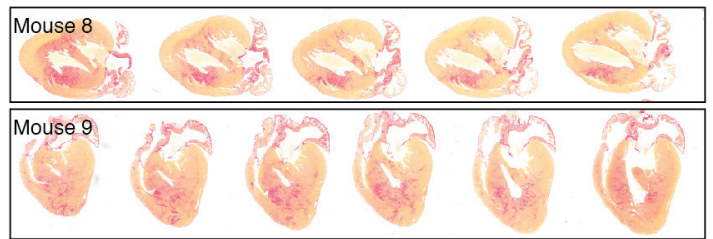

Mouse 10

(3.

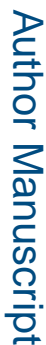

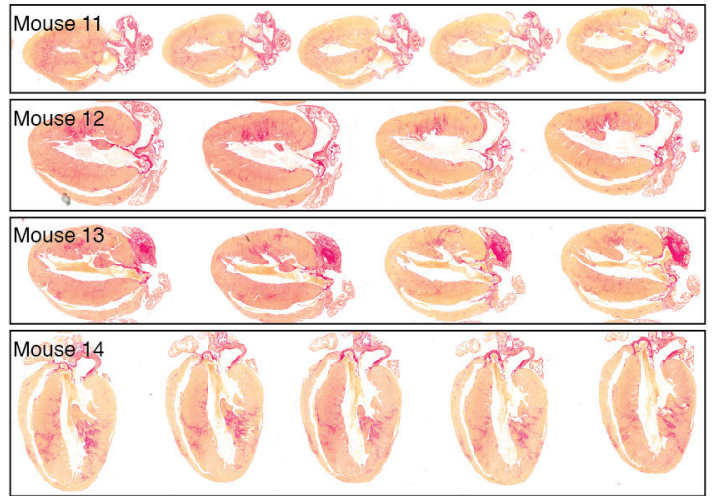

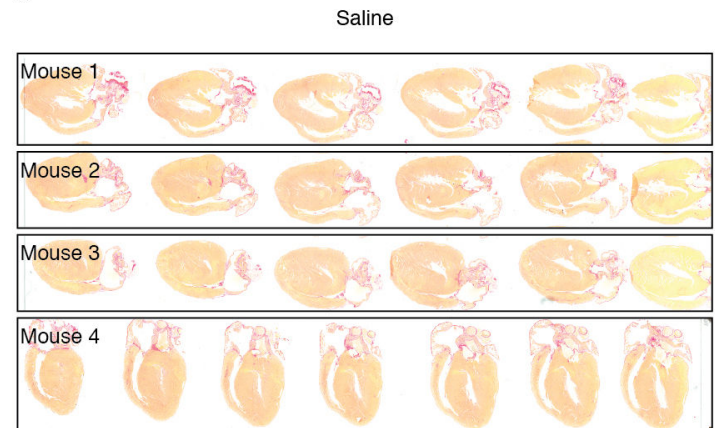
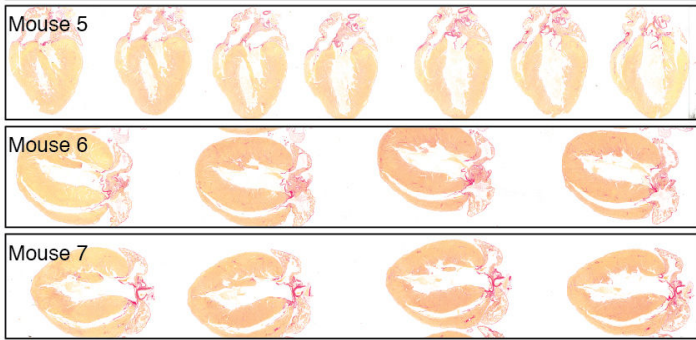

d $\quad$ Angll/PE + FAP CAR T Cells

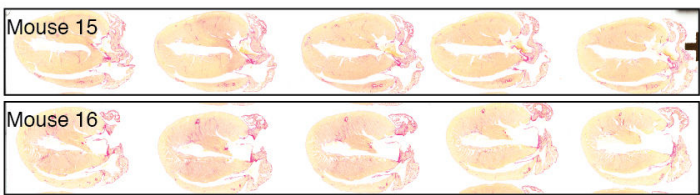

Mouse 17
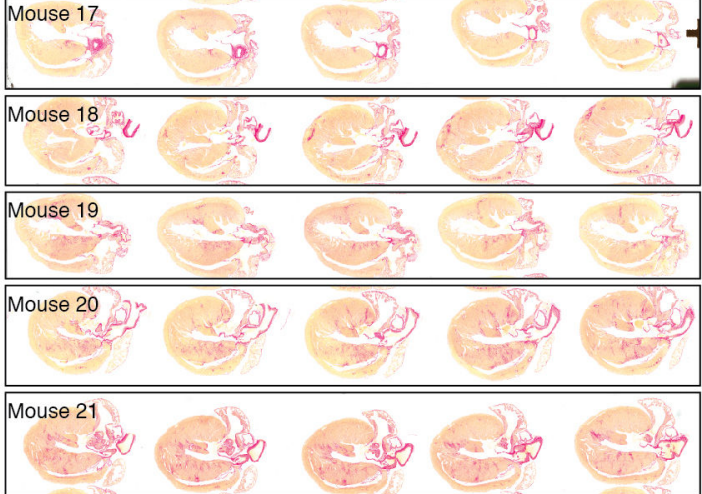

Extended Data Fig. 4.

FAP CAR T cells infiltrate the heart and reduce cardiac fibrosis.

(a) Immunohistochemistry for Fap (red) and GFP (green) on the left ventricular free wall of mouse heart coronal sections. WT C57B1/6 mice were treated with (right) or without (left) AngII/PE for 1 week, injected with FAP-GFP CAR T cells and sacrificed 1 day later. FAPGFP CAR T cells co-localize with FAP expressing cells (arrowheads, bottom). (b-d) PicroSirus Red staining of hearts from 21 individual mice (\#1-21) treated for 4 weeks with either saline (b), AngII/PE (c), or AngII/PE + FAP CAR T cells (d) to assess for fibrosis (red). 
Representative images of 2 independent experiments with similar results. Scale bars = $100 \mu \mathrm{m}$. 
EDV

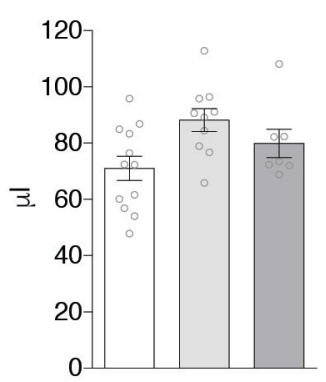

SV

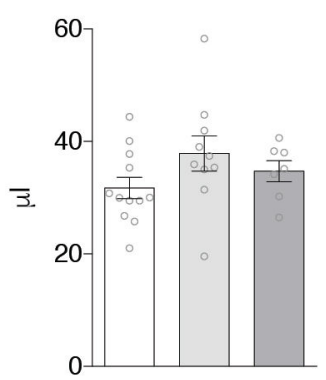

IVSd

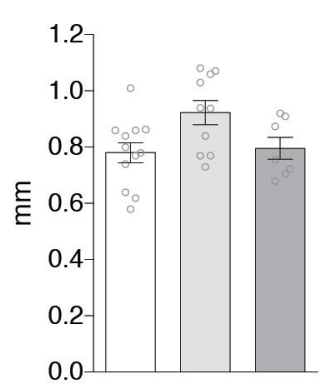

ESV

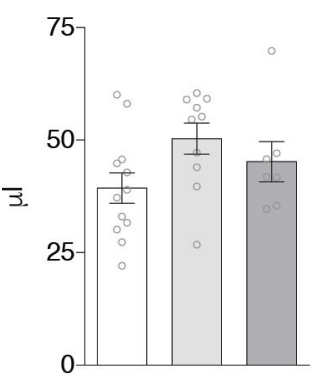

$\mathrm{CO}$

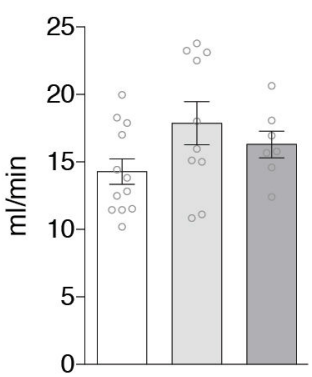

IVSs

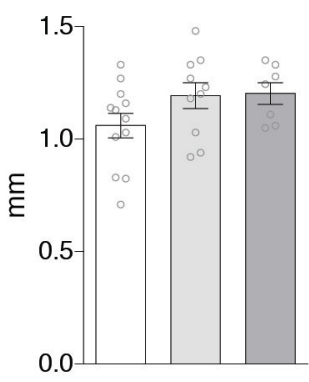

LVAENDd

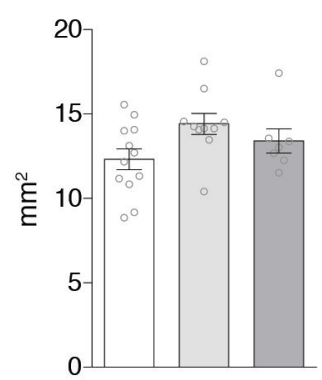

LVLd

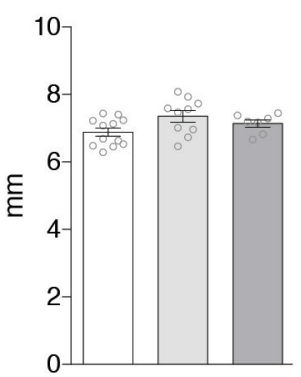

LVIDd

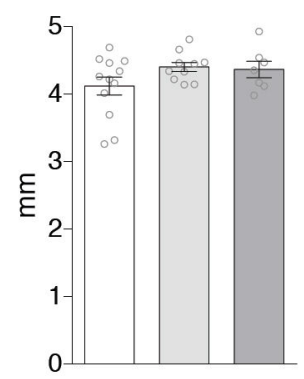

LVAENDS

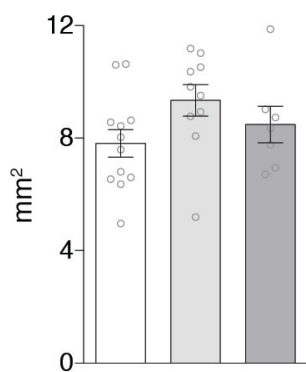

LVLS

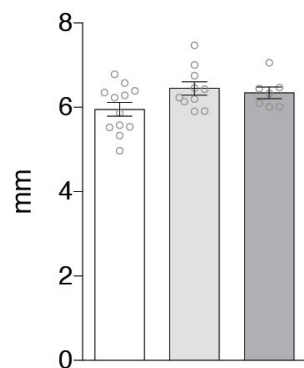

LVIDs

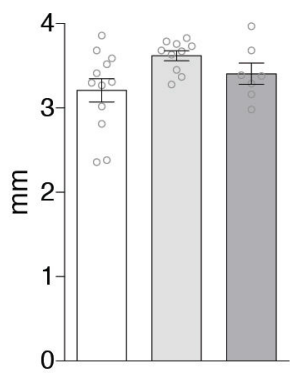

LVAEpid

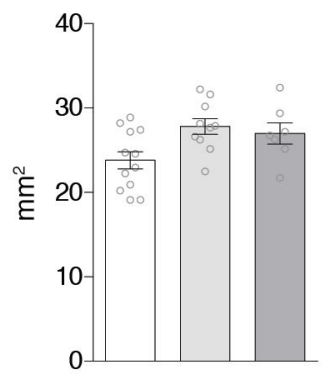

$E / E^{\prime}$

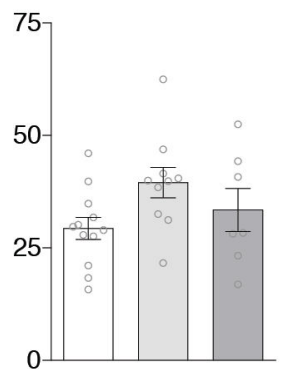

Extended Data Fig. 5.

Echocardiography after injury and treatment.

Results from echocardiogram examination of C57B1/6 mice treated for 4 weeks with either saline, AngII/PE, or AngII/PE + FAP CAR T cells. $(n=12,10$, and 7 biologically independent animals, respectively) FS = Fractional shortening; HR = heart rate; BW = body weight; LVAEpid = Left ventricular epicardial area (diastole); LVAENDd $=$ Left ventricular endocardial area (diastole); LVAENDs = Left ventricular endocardial area (systole); LVLd = Left ventricular endocardial length (diastole); LVLs = Left ventricular endocardial length (systole); EDV = End diastolic volume; ESV = End systolic volume; SV = Stroke volume; $\mathrm{CO}=$ Cardiac output; IVSd = Interventricular septal end diastole; IVSs = Interventricular septal end systole; LVIDd = Left ventricular internal diameter end diastole; LVIDs = Left ventricular internal diameter end systole; $\mathrm{MV} \mathrm{E}=$ Early ventricular filling velocity; $\mathrm{E} / \mathrm{E}^{\prime}$ = ratio of mitral peak velocity of early filling (E) to early diastolic mitral annular velocity (E'). All graphs display mean \pm SEM. 

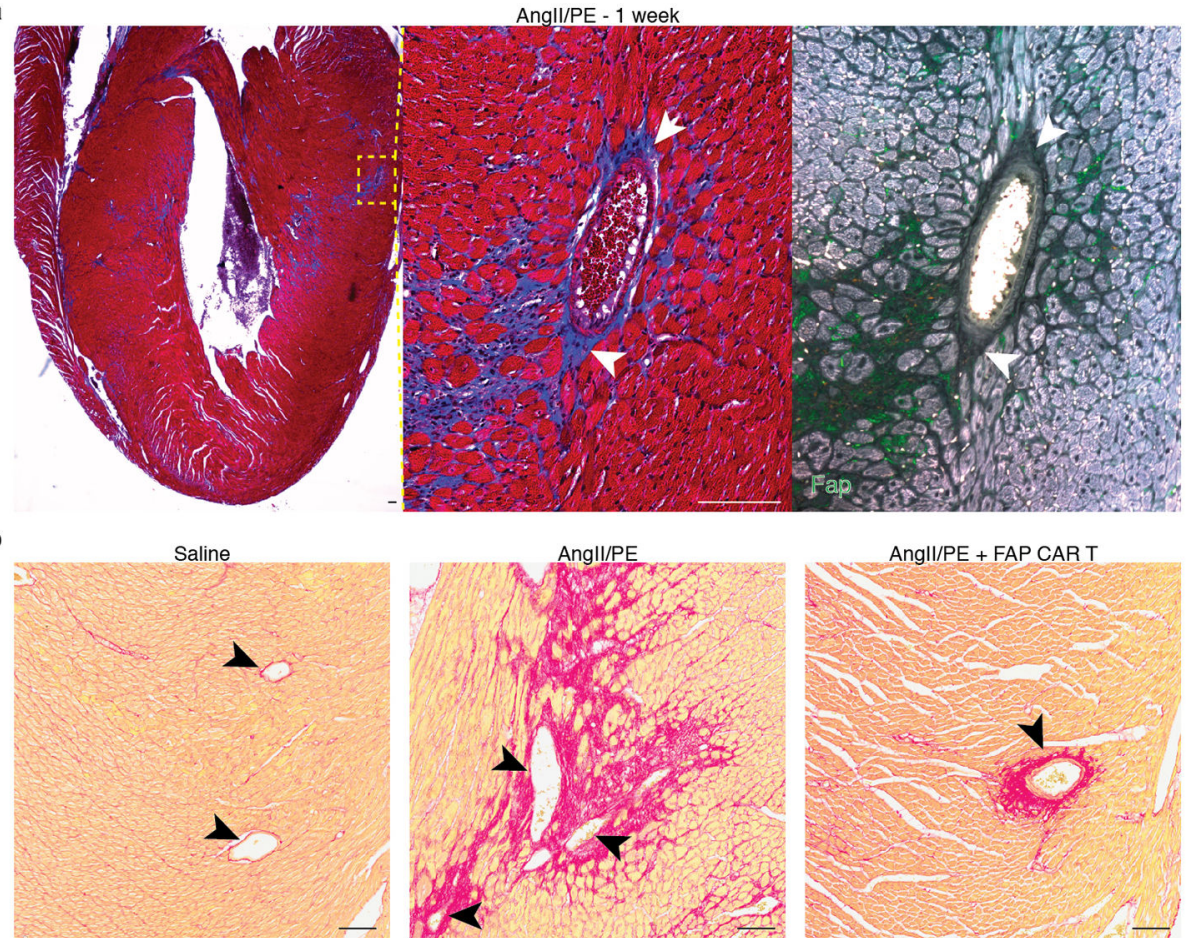

Angll/PE + FAP CAR T
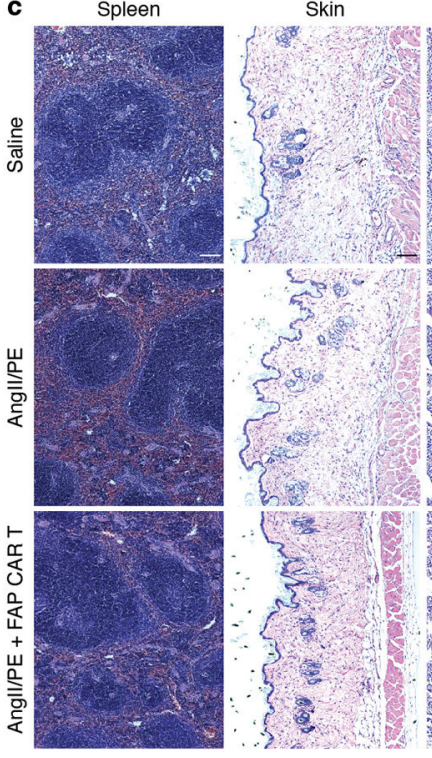

Testis

Lung

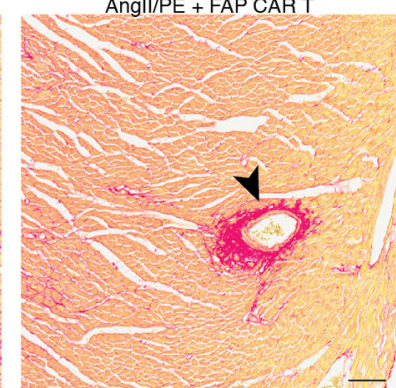

Extended Data Fig. 6.

FAP CAR T treatment does not affect perivascular fibrosis or other organs.

(a) Masson's trichrome stain (blue; left, center) and FAP immunohistochemistry (green; right) on adjacent heart coronal sections 1 week after commencement of continuous

AngII/PE treatment. FAP expression in present in interstitial, but not perivascular fibroblast (white arrowheads). Centered on vessel from Fig. 2c. Representative images of 2 independent experiments with similar results. (b) Picro-Sirius Red staining for perivascular fibrosis (black arrowheads, red) on heart coronal sections from mice treated for 4 weeks with 
either saline, AngII/PE, or AngII/PE + FAP CAR T cells. Representative images of 2 independent experiments with similar results. (c) H\&E staining of various tissue sections from mice treated for 4 weeks with either saline, AngII/PE, or AngII/PE + FAP CAR T cells. Representative images of 3 independent experiments with similar results. Scale bars $=$ $100 \mu \mathrm{m}$. 

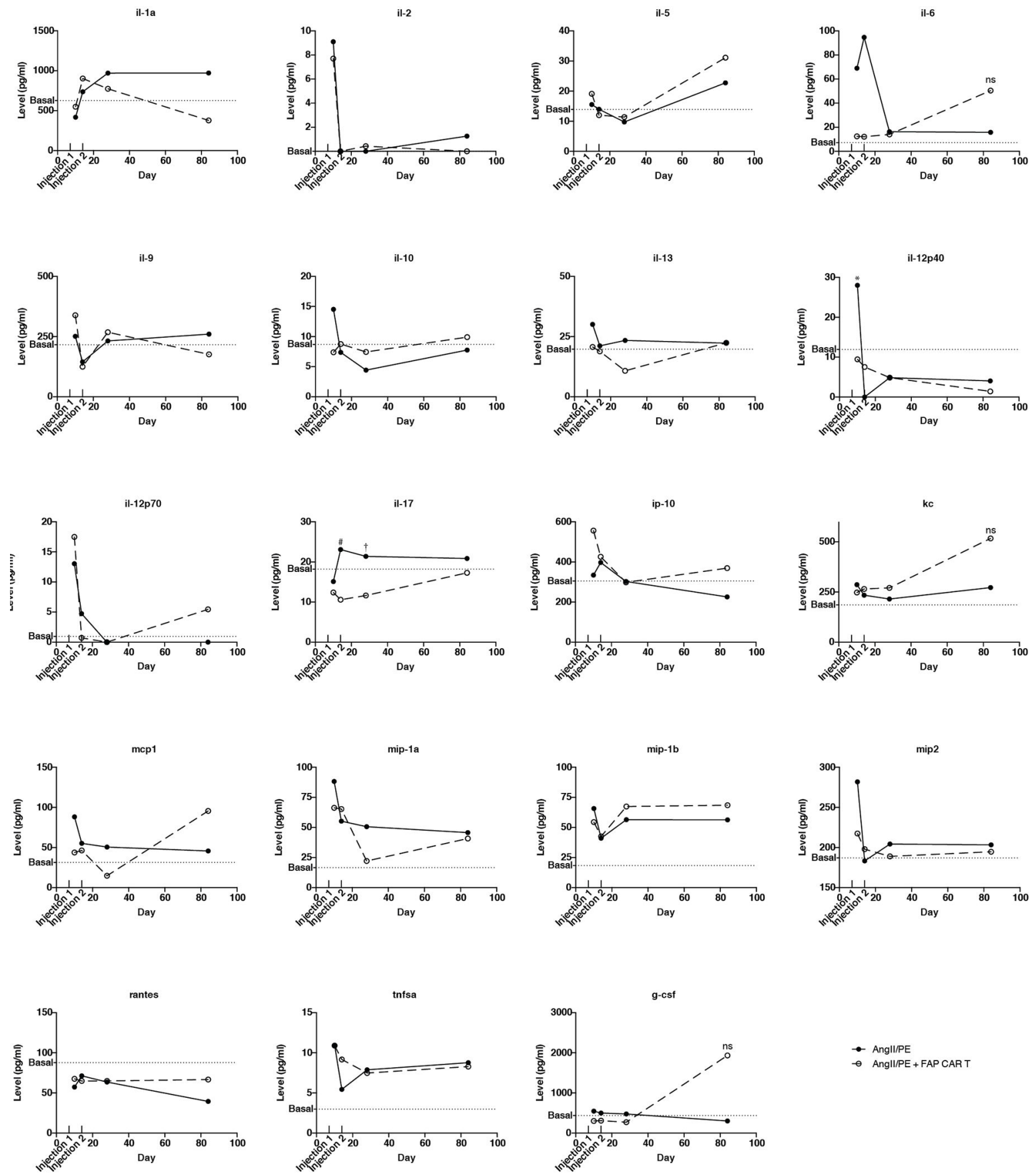

- AngIIPE

$\rightarrow$ Angll/PE + FAP CAR

Extended Data Fig. 7.

Long term serum cytokine levels after FAP CAR T cell treatment.

Serum cytokine levels in mice treated with either AngII/PE or AngII/PE + FAP CAR T cells over 12 weeks. FAP CAR T cells were injected at 1 and 2 weeks as indicated. Levels were assessed at 10 days, 2 weeks, 4 weeks, and 12 weeks. Basal levels were determined by the average cytokine levels of 3 untreated mice. Infg and il-4 were below the detection limit in all conditions. ${ }^{*} P=0.019, \# P=0.035, \dagger P=0.045$ (Two-tailed unpaired t-test, $\mathrm{n}=3$ biologically independent animals per condition). 
a
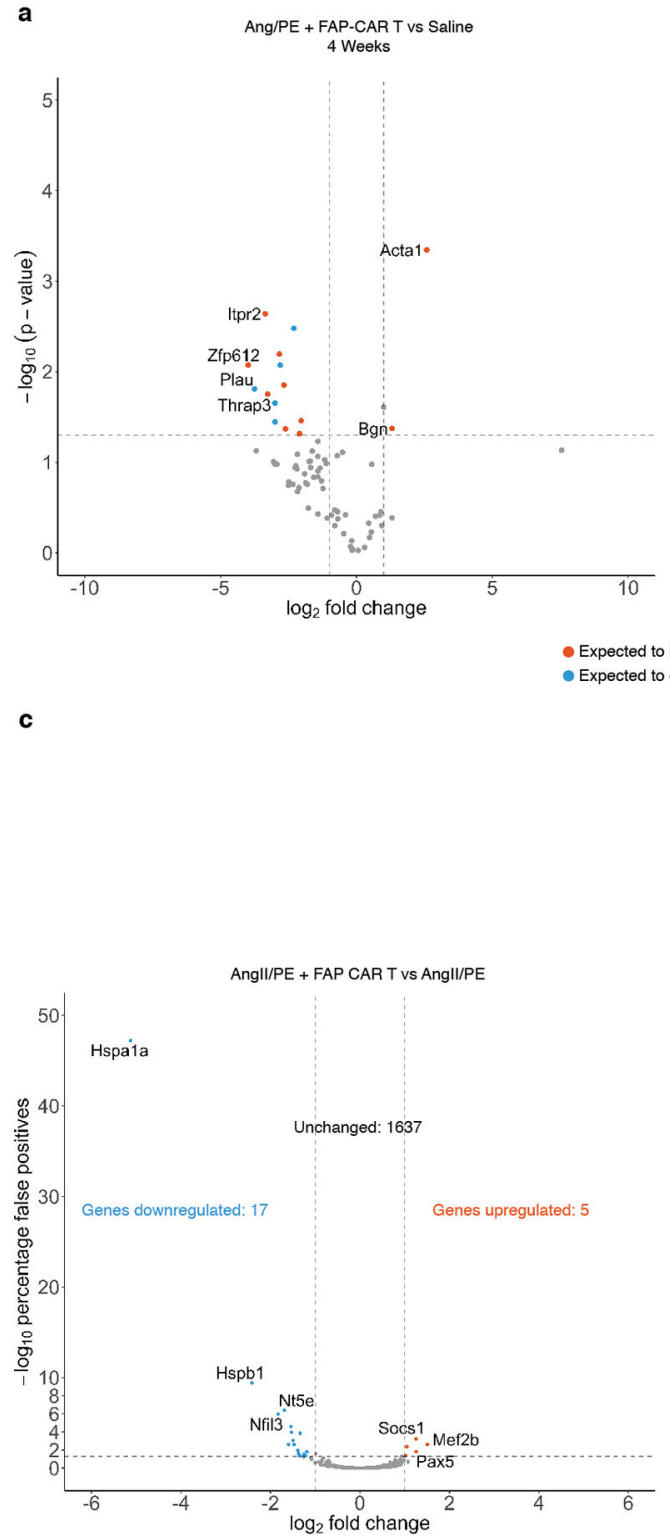

c

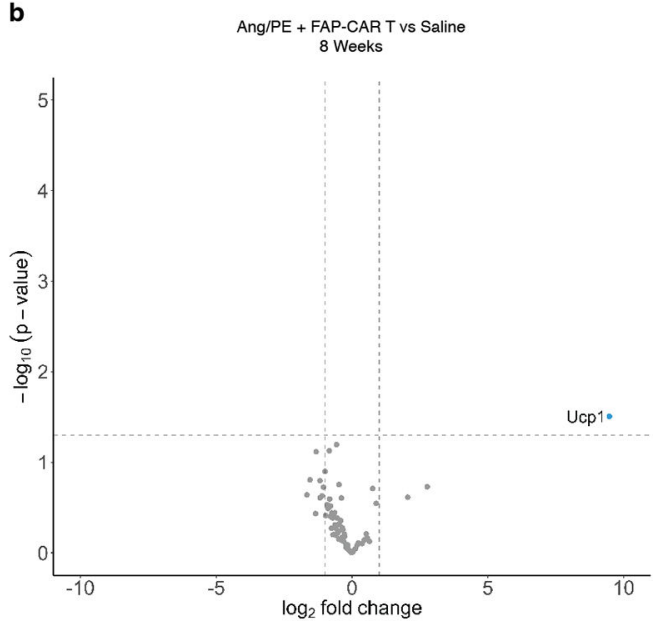
- Expected to increase in cardiotoxicity

d
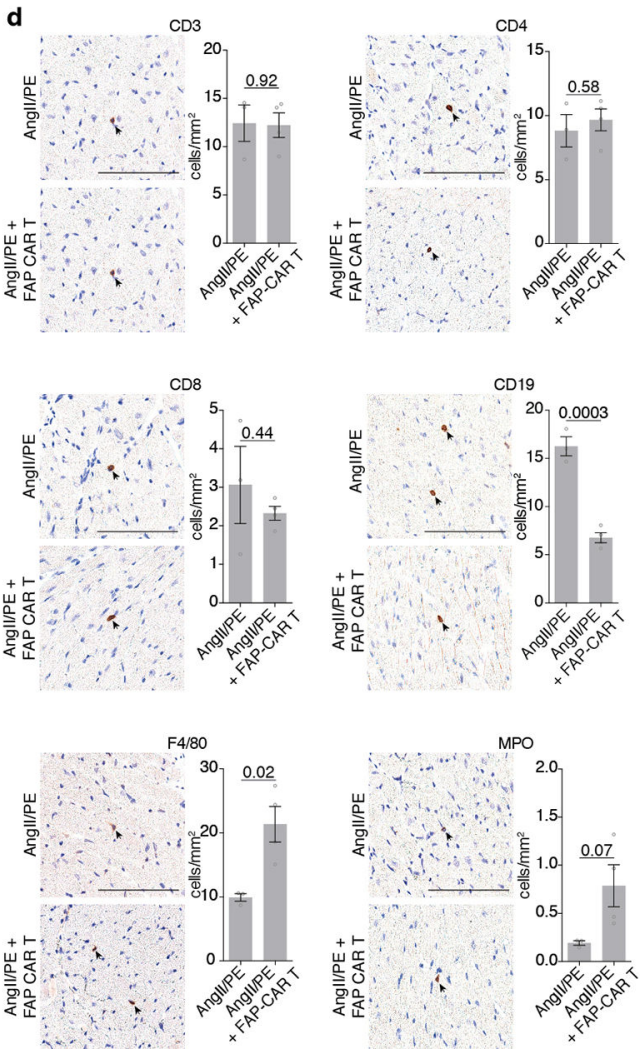

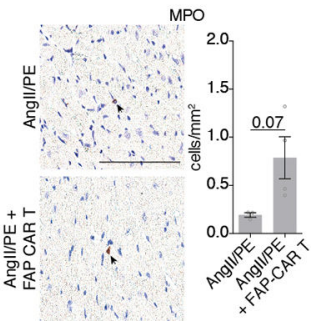

Extended Data Fig. 8.

Cardiotoxicity, inflammation, and immune assessments after FAP CAR T cell transfer. (a) Volcano plot showing the differential expression of genes known to be modified in cardiotoxicity in the hearts of mice treated with either AngII/PE + FAP CAR T cells or a saline control at 4 weeks. Statistically significant changes are marked to indicate if genes are expected to increase (orange) or decrease (blue) in the setting of cardiotoxicity. (b) Differential expression of the same conditions in (a) at 8 weeks. (a, b-Welch's two-sided test, $n=3$ biologically independent animals per condition) (c) Volcano plot showing the 
differential expression of 1659 immune- and inflammation-related genes from hearts of mice treated with AngII/PE +FAP CAR T cells or AngII/PE at 4 weeks. 22 genes were differentially expressed between the conditions. ( $\mathrm{n}=3$ mice per condition) (d) Photomicrographs and quantification (mean \pm SEM) of immune cell (arrowheads) residency of the left ventricle at 4 weeks with either AngII/PE or AngII/PE + FAP CAR T cell treatment. (Two-tailed unpaired t-test, $\mathrm{n}=3,4$ biologically independent animals, respectively). Scale bars $=100 \mu \mathrm{m}$. 


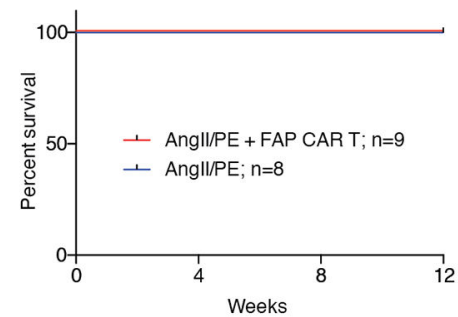

b

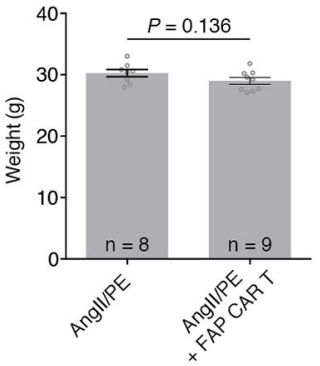

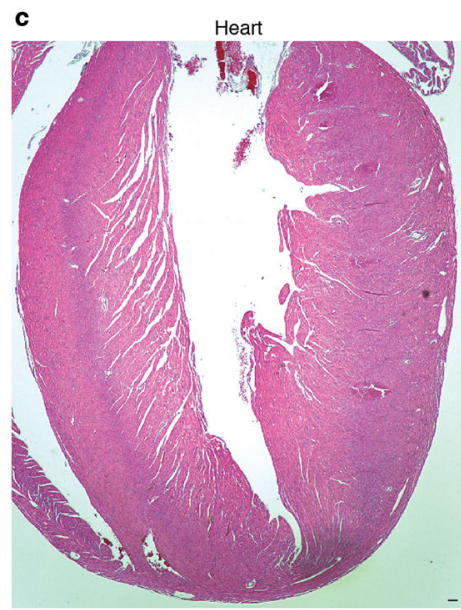
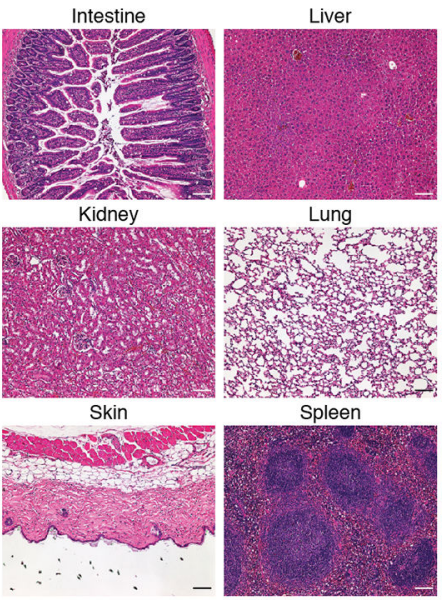

Pancreas
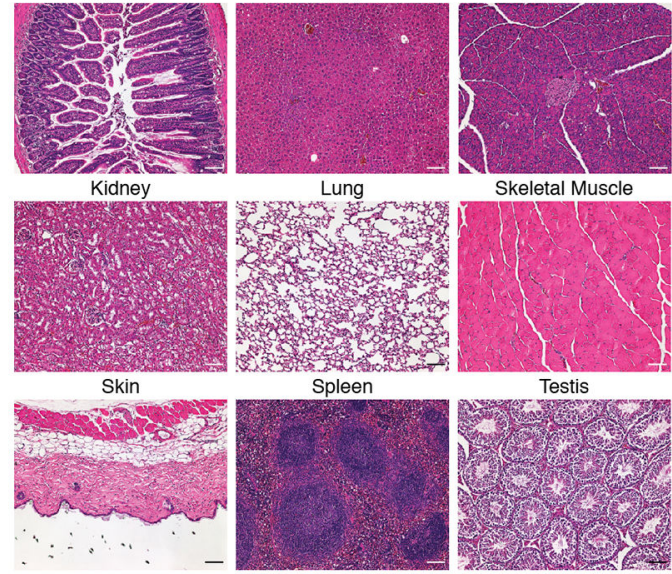

Skeletal Muscle

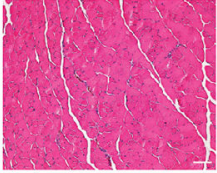

Testis
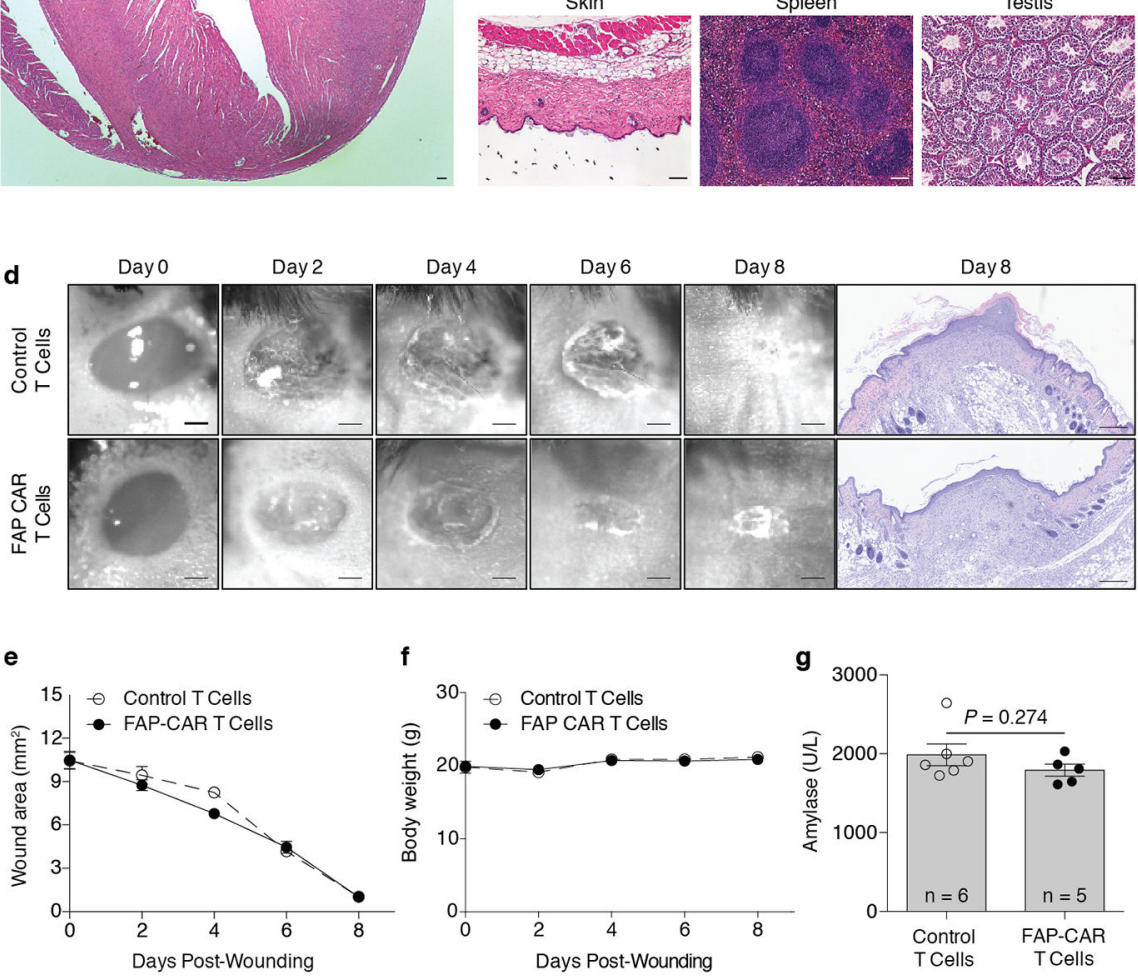

Extended Data Fig. 9.

Assessment of safety and toxicity following FAP CAR T cell transfer.

(a) Kaplan-Meier survival curve of mice either treated with AngII/PE or AngII/PE + FAP

CAR T cells out to 12 weeks. (b) Body weight measurements at 12 weeks. (Two-tailed unpaired t-test) (c) H\&E of sections of heart and organs/tissue at 12 weeks from a mouse treated with AngII/PE + FAP CAR T cells. Representative images of 3 independent animals with similar results. Scale bars $=100 \mu \mathrm{m}$. (d) Photomicrographs and H\&E sections of a healing wound over 8 days in mice treated with either FAP CAR or control T cells 
immediately and 3 days after wounding. (e) Quantification of wound area and measurements of body weight (f). (g) Serum levels of amylase at day 8 to test pancreatic toxicity. Scale bars $=1 \mathrm{~mm}$ (wounds) and $250 \mu \mathrm{m}$ (H\&E sections). All graphs display mean \pm SEM.

\section{Supplementary Material}

Refer to Web version on PubMed Central for supplementary material.

\section{Acknowledgements}

We thank Jeffery Molkentin for kindly sharing the Perisostin $M C M_{\text {mice }}$ with us. We thank Andrea Stout for help with imaging. We thank Daniel Martinez for help with image analysis. We thank Marielle Scherrer-Crosbie for guidance with echocardiogram procedure and interpretation. We thank Foteini Mourkioti for kindly sharing the $m d x / m T R$ mice us. We thank Enrico Radaelli for help with IHC. We thank the University of Pennsylvania Diabetes Research Center (DRC) for the use of the RIA Biomarker Core (P30-DK19525). This research was supported by NIH R35 HL140018, T32 HL007843-22, the Cotswold Foundation, and the WW Smith Endowed Chair to J.A.E

\section{References}

1. Travers JG, Kamal FA, Robbins J, Yutzey KE \& Blaxall BC Cardiac Fibrosis: The Fibroblast Awakens. Circ Res 118, 1021-1040, doi:10.1161/CIRCRESAHA.115.306565 (2016). [PubMed: 26987915]

2. Kong P, Christia P \& Frangogiannis NG The pathogenesis of cardiac fibrosis. Cell Mol Life Sci 71, 549-574, doi:10.1007/s00018-013-1349-6 (2014). [PubMed: 23649149]

3. Fang L, Murphy AJ \& Dart AM A Clinical Perspective of Anti-Fibrotic Therapies for Cardiovascular Disease. Front Pharmacol 8, 186, doi:10.3389/fphar.2017.00186 (2017). [PubMed: 28428753]

4. Ottaviano FG \& Yee KO Communication signals between cardiac fibroblasts and cardiac myocytes. J Cardiovasc Pharmacol 57, 513-521, doi:10.1097/FJC.0b013e31821209ee (2011). [PubMed: 21297490]

5. Fan Z \& Guan J Antifibrotic therapies to control cardiac fibrosis. Biomater Res 20, 13, doi:10.1186/ s40824-016-0060-8 (2016). [PubMed: 27226899]

6. Lam CSP, Voors AA, de Boer RA, Solomon SD \& van Veldhuisen DJ Heart failure with preserved ejection fraction: from mechanisms to therapies. Eur Heart J, doi:10.1093/eurheartj/ehy301 (2018).

7. Kaur $\mathrm{H}$ et al. Targeted Ablation of Periostin-Expressing Activated Fibroblasts Prevents Adverse Cardiac Remodeling in Mice. Circ Res 118, 1906-1917, doi:10.1161/CIRCRESAHA.116.308643 (2016). [PubMed: 27140435]

8. Kanisicak $\mathrm{O}$ et al. Genetic lineage tracing defines myofibroblast origin and function in the injured heart. Nat Commun 7, 12260, doi:10.1038/ncomms12260 (2016). [PubMed: 27447449]

9. Schmitt TM, Ragnarsson GB \& Greenberg PD T cell receptor gene therapy for cancer. Hum Gene Ther 20, 1240-1248, doi:10.1089/hum.2009.146 (2009). [PubMed: 19702439]

10. June CH, O'Connor RS, Kawalekar OU, Ghassemi S \& Milone MC CAR T cell immunotherapy for human cancer. Science 359, 1361-1365, doi:10.1126/science.aar6711 (2018). [PubMed: 29567707]

11. Mullard A FDA approves first CAR T therapy. Nat Rev Drug Discov 16, 669, doi:10.1038/nrd. 2017.196 (2017).

12. Ghobadi A Chimeric antigen receptor T cell therapy for non-Hodgkin lymphoma. Curr Res Transl Med 66, 43-49, doi:10.1016/j.retram.2018.03.005 (2018). [PubMed: 29655961]

13. June CH \& Sadelain M Chimeric Antigen Receptor Therapy. N Engl J Med 379, 64-73, doi: 10.1056/NEJMra1706169 (2018). [PubMed: 29972754]

14. Lim WA \& June CH The Principles of Engineering Immune Cells to Treat Cancer. Cell 168, 724740, doi:10.1016/j.cell.2017.01.016 (2017). [PubMed: 28187291] 
15. Sandhu U et al. Strict control of transgene expression in a mouse model for sensitive biological applications based on RMCE compatible ES cells. Nucleic Acids Res 39, e1, doi:10.1093/nar/ gkq868 (2011). [PubMed: 20935052]

16. Cebula $\mathrm{M}$ et al. An inducible transgenic mouse model for immune mediated hepatitis showing clearance of antigen expressing hepatocytes by CD8+ T cells. PLoS One 8, e68720, doi:10.1371/ journal.pone.0068720 (2013). [PubMed: 23869228]

17. Hogquist KA et al. T cell receptor antagonist peptides induce positive selection. Cell 76, 17-27 (1994). [PubMed: 8287475]

18. Clarke SR et al. Characterization of the ovalbumin-specific TCR transgenic line OT-I: MHC elements for positive and negative selection. Immunol Cell Biol 78, 110-117, doi:10.1046/j. 1440-1711.2000.00889.x (2000). [PubMed: 10762410]

19. Ivey MJ \& Tallquist MD Defining the Cardiac Fibroblast. Circ J 80, 2269-2276, doi:10.1253/ circj.CJ-16-1003 (2016). [PubMed: 27746422]

20. Tallquist MD \& Molkentin JD Redefining the identity of cardiac fibroblasts. Nat Rev Cardiol 14, 484-491, doi:10.1038/nrcardio.2017.57 (2017). [PubMed: 28436487]

21. Scanlan MJ et al. Molecular cloning of fibroblast activation protein alpha, a member of the serine protease family selectively expressed in stromal fibroblasts of epithelial cancers. Proc Natl Acad Sci U S A 91, 5657-5661 (1994). [PubMed: 7911242]

22. Rettig WJ et al. Cell-surface glycoproteins of human sarcomas: differential expression in normal and malignant tissues and cultured cells. Proc Natl Acad Sci U S A 85, 3110-3114 (1988). [PubMed: 2896356]

23. Niedermeyer $\mathrm{J}$ et al. Mouse fibroblast activation protein: molecular cloning, alternative splicing and expression in the reactive stroma of epithelial cancers. Int J Cancer 71, 383-389 (1997). [PubMed: 9139873]

24. Tillmanns $\mathbf{J}$ et al. Fibroblast activation protein alpha expression identifies activated fibroblasts after myocardial infarction. J Mol Cell Cardiol 87, 194-203, doi:10.1016/j.yjmcc.2015.08.016 (2015). [PubMed: 26319660]

25. Wang LC et al. Targeting fibroblast activation protein in tumor stroma with chimeric antigen receptor $\mathrm{T}$ cells can inhibit tumor growth and augment host immunity without severe toxicity. Cancer Immunol Res 2, 154-166, doi:10.1158/2326-6066.CIR-13-0027 (2014). [PubMed: 24778279]

26. Kakarla $\mathrm{S}$ et al. Antitumor effects of chimeric receptor engineered human T cells directed to tumor stroma. Mol Ther 21, 1611-1620, doi:10.1038/mt.2013.110 (2013). [PubMed: 23732988]

27. Lo A et al. Tumor-Promoting Desmoplasia Is Disrupted by Depleting FAP-Expressing Stromal Cells. Cancer Res 75, 2800-2810, doi:10.1158/0008-5472.CAN-14-3041 (2015). [PubMed: 25979873]

28. Schuberth PC et al. Treatment of malignant pleural mesothelioma by fibroblast activation proteinspecific re-directed T cells. J Transl Med 11, 187, doi:10.1186/1479-5876-11-187 (2013). [PubMed: 23937772]

29. Petrausch $U$ et al. Re-directed $T$ cells for the treatment of fibroblast activation protein (FAP)positive malignant pleural mesothelioma (FAPME-1). BMC Cancer 12, 615, doi: 10.1186/1471-2407-12-615 (2012). [PubMed: 23259649]

30. Govindaraju P, Todd L, Shetye S, Monslow J \& Pure E CD44-dependent inflammation, fibrogenesis, and collagenolysis regulates extracellular matrix remodeling and tensile strength during cutaneous wound healing. Matrix Biol 75-76, 314-330, doi:10.1016/j.matbio.2018.06.004 (2019).

31. Croft AP et al. Distinct fibroblast subsets drive inflammation and damage in arthritis. Nature, doi: 10.1038/s41586-019-1263-7 (2019).

32. Fischbach MA, Bluestone JA \& Lim WA Cell-based therapeutics: the next pillar of medicine. Sci Transl Med 5, 179ps177, doi:10.1126/scitranslmed.3005568 (2013).

33. Tran $\mathrm{E}$ et al. Immune targeting of fibroblast activation protein triggers recognition of multipotent bone marrow stromal cells and cachexia. J Exp Med 210, 1125-1135, doi:10.1084/jem.20130110 (2013). [PubMed: 23712432] 
34. Cameron BJ et al. Identification of a Titin-derived HLA-A1-presented peptide as a cross-reactive target for engineered MAGE A3-directed T cells. Sci Transl Med 5, 197ra103, doi:10.1126/ scitranslmed.3006034 (2013).

35. Linette GP et al. Cardiovascular toxicity and titin cross-reactivity of affinity-enhanced T cells in myeloma and melanoma. Blood 122, 863-871, doi:10.1182/blood-2013-03-490565 (2013). [PubMed: 23770775]

36. Sun S, Hao H, Yang G, Zhang Y \& Fu Y Immunotherapy with CAR-Modified T Cells: Toxicities and Overcoming Strategies. J Immunol Res 2018, 2386187, doi:10.1155/2018/2386187 (2018). [PubMed: 29850622]

\section{Methods References}

37. Kanisicak $\mathrm{O}$ et al. Genetic lineage tracing defines myofibroblast origin and function in the injured heart. Nat Commun 7, 12260, doi:10.1038/ncomms12260 (2016). [PubMed: 27447449]

38. Sandhu U et al. Strict control of transgene expression in a mouse model for sensitive biological applications based on RMCE compatible ES cells. Nucleic Acids Res 39, e1, doi:10.1093/nar/ gkq868 (2011). [PubMed: 20935052]

39. Cebula $\mathrm{M}$ et al. An inducible transgenic mouse model for immune mediated hepatitis showing clearance of antigen expressing hepatocytes by CD8+ T cells. PLoS One 8, e68720, doi:10.1371/ journal.pone.0068720 (2013). [PubMed: 23869228]

40. Ochel A et al. Effective intrahepatic CD8+ T-cell immune responses are induced by low but not high numbers of antigen-expressing hepatocytes. Cell Mol Immunol 13, 805-815, doi:10.1038/ cmi.2015.80 (2016). [PubMed: 26412123]

41. Mourkioti F et al. Role of telomere dysfunction in cardiac failure in Duchenne muscular dystrophy. Nat Cell Biol 15, 895-904, doi:10.1038/ncb2790 (2013). [PubMed: 23831727]

42. Wang LC et al. Targeting fibroblast activation protein in tumor stroma with chimeric antigen receptor $\mathrm{T}$ cells can inhibit tumor growth and augment host immunity without severe toxicity. Cancer Immunol Res 2, 154-166, doi:10.1158/2326-6066.CIR-13-0027 (2014). [PubMed: 24778279]

43. Newick K et al. Augmentation of CAR T-cell Trafficking and Antitumor Efficacy by Blocking Protein Kinase A Localization. Cancer Immunol Res 4, 541-551, doi: 10.1158/2326-6066.CIR-15-0263 (2016). [PubMed: 27045023]

44. Dipla K, Mattiello JA, Jeevanandam V, Houser SR \& Margulies KB Myocyte recovery after mechanical circulatory support in humans with end-stage heart failure. Circulation 97, 2316-2322, doi:10.1161/01.cir.97.23.2316 (1998). [PubMed: 9639375]

45. Chen CY et al. Suppression of detyrosinated microtubules improves cardiomyocyte function in human heart failure. Nat Med 24, 1225-1233, doi:10.1038/s41591-018-0046-2 (2018). [PubMed: 29892068]

46. Dobin A et al. STAR: ultrafast universal RNA-seq aligner. Bioinformatics 29, 15-21, doi:10.1093/ bioinformatics/bts635 (2013). [PubMed: 23104886]

47. Law CW, Chen Y, Shi W \& Smyth GK voom: Precision weights unlock linear model analysis tools for RNA-seq read counts. Genome Biol 15, R29, doi:10.1186/gb-2014-15-2-r29 (2014). [PubMed: 24485249]

48. Love MI, Huber W \& Anders S Moderated estimation of fold change and dispersion for RNA-seq data with DESeq2. Genome Biol 15, 550, doi:10.1186/s13059-014-0550-8 (2014). [PubMed: 25516281]

49. Benjamini Y \& Hochberg Y Controlling the False Discovery Rate - a Practical and Powerful Approach to Multiple Testing. J R Stat Soc B 57, 289-300 (1995). 


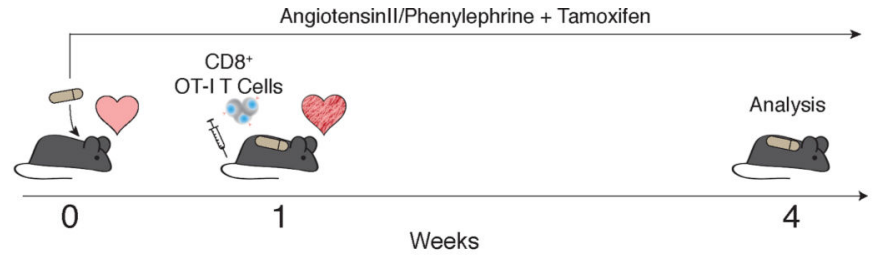

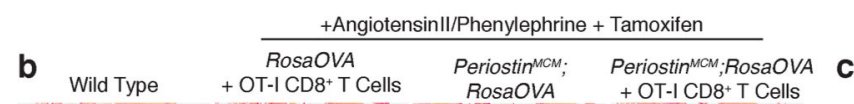

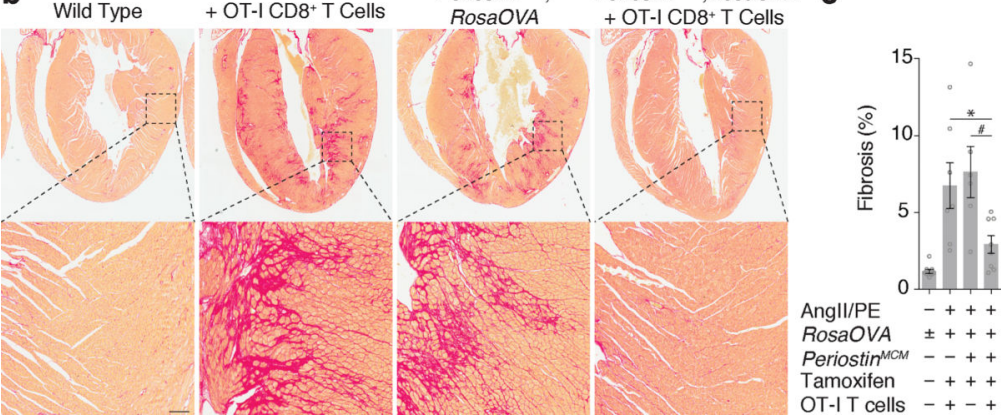

Fig. 1.

Redirected T cell can ablate cardiac fibroblasts. (a) Schematic representation of cardiac injury and $\mathrm{T}$ cell mediated cardiac fibroblast ablation. Mice were continuously administered angiotensin II and phenylephrine via osmotic pump to induce cardiac injury and fibrosis, and injected with tamoxifen to trigger Cre-mediated OVA expression in the CFs. CD8 ${ }^{+}$OT-I T cells were adoptively transferred 1 week after pump implantation when fibrosis was already established, and mice were sacrificed 4 weeks post-implantation for analysis. (b) PicroSirius Red staining of heart coronal sections to evaluate the level of fibrosis (red). Higher magnification of left ventricular fibrosis (bottom). (c) Quantification of ventricular fibrosis (mean $\pm \mathrm{SEM}$ ). ${ }^{*} P=0.0492, \# P=0.0157$, (one-way ANOVA between groups $P=0.0001$; post-hoc multiple comparisons, Tukey's test, $n=10,7,6,8$ biologically independent animals, respectively). Scale bars $=100 \mu \mathrm{m}$. 


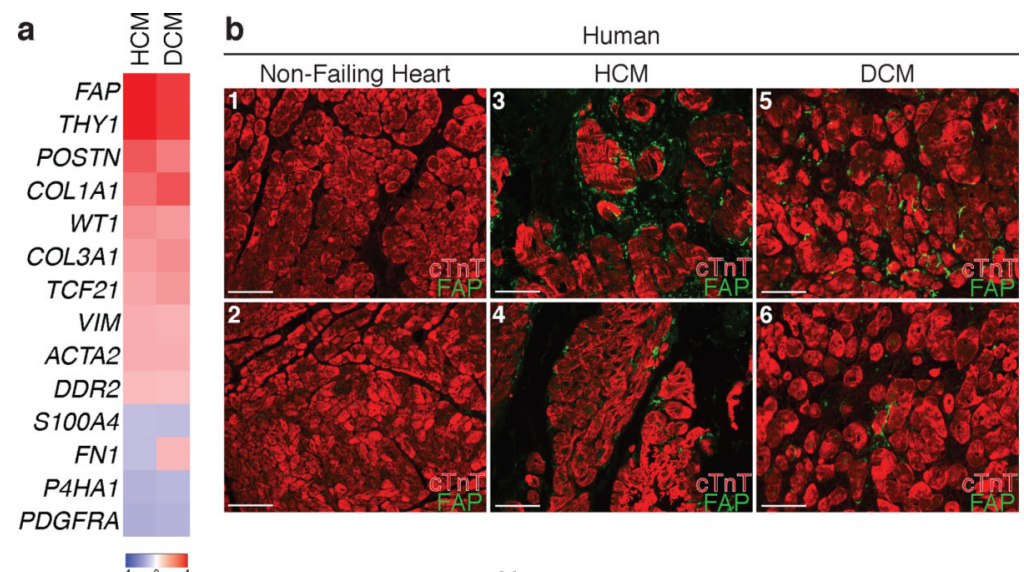

C

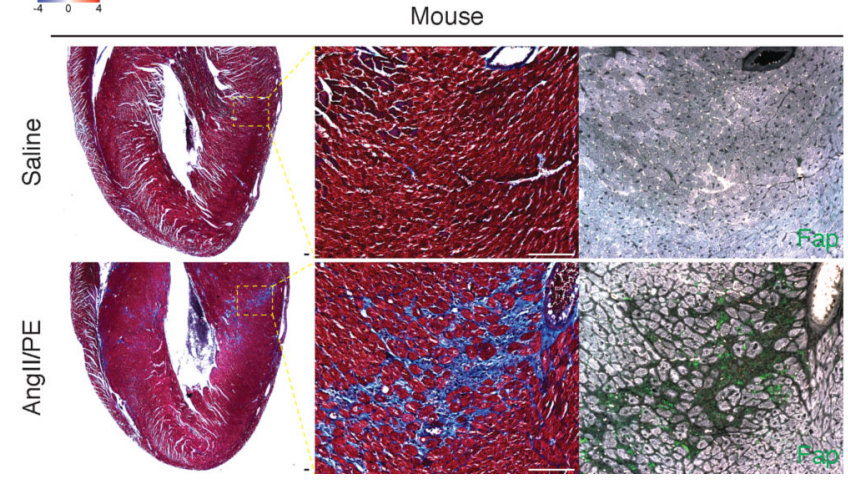

Fig. 2.

Human cardiac fibroblast targets in disease. (a) Heat map of cardiac fibroblast gene expression changes (fold change) in patients with hypertrophic cardiomyopathy (HCM) and dilated cardiomyopathy (DCM) when compared with non-failing hearts (NF). ( $\mathrm{n}=122(\mathrm{NF})$, 27 (HCM), 89 (DCM)) Differential gene expression was performed using a linear model. (b) Immunohistochemistry for FAP (green) and cardiac Troponin (cardiomyocytes, red) expression in left ventricular free-wall sections from 6 individual human samples of NF $(\# 1,2), \operatorname{HCM}(\# 3,4)$, and DCM $(\# 5,6)$. Representative images of 2 independent experiments with similar results. (c) Masson's trichrome stain for fibrosis (blue; left, center) and immunohistochemistry for Fap (green; right) in adjacent WT mouse coronal heart sections 1 week after continuous saline (top) or angiotensin II/phenylephrine (bottom) treatment. Representative images of 2 independent experiments with similar results ( $n=7$ biologically independent animals) (inset; left ventricle). Scale bars $=100 \mu \mathrm{m}$. 

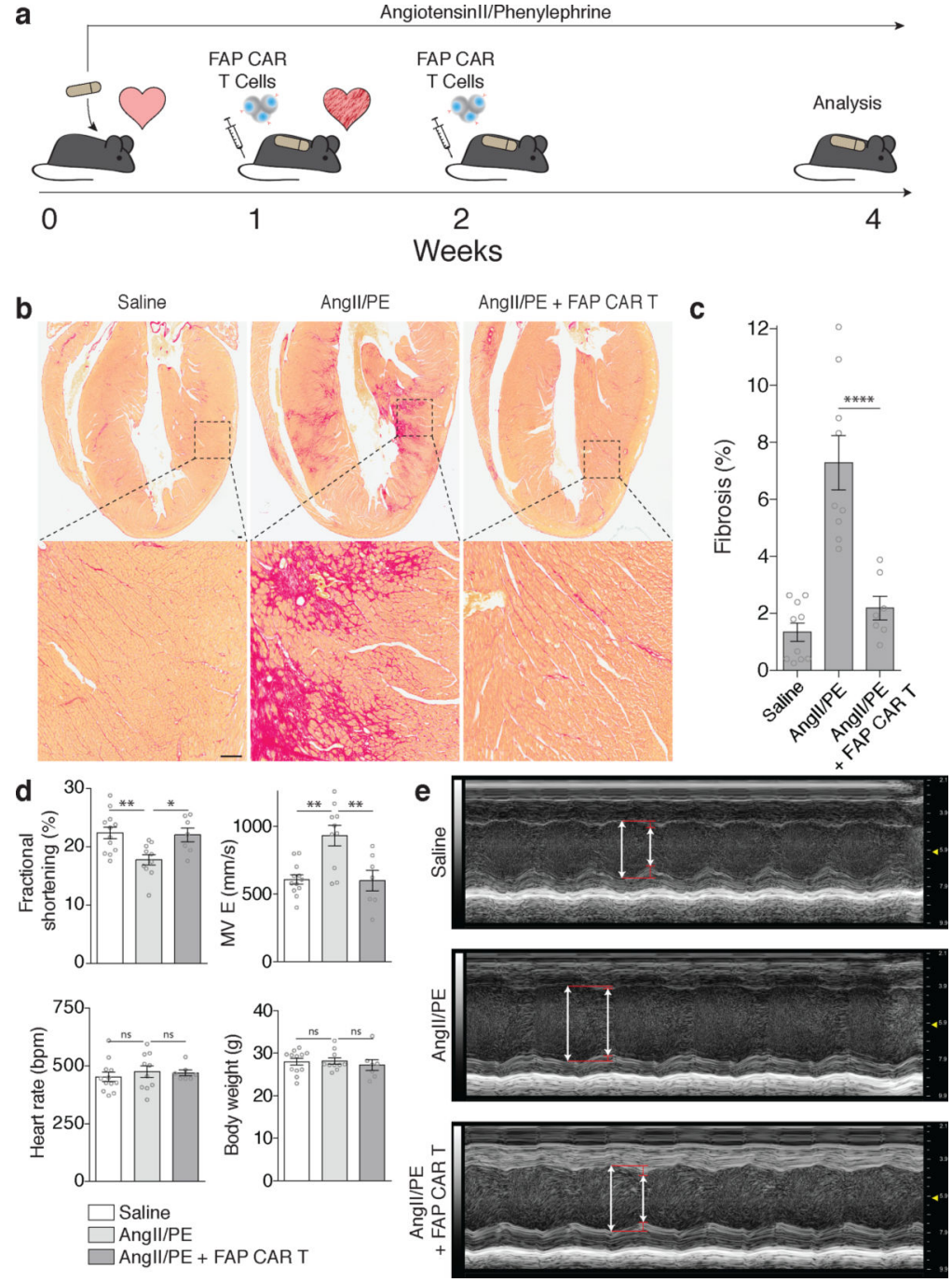

Fig. 3.

FAP CAR T cells can target cardiac fibrosis. (a) Schematic diagram of experiments for FAP CAR T-cell targeting of cardiac fibroblast. C57BL/6 mice were continuously administered angiotensin II and phenylephrine via osmotic pump to induce cardiac injury and fibrosis. FAP CAR T cells were adoptively transferred 1 and 2 weeks after pump implantation when fibrosis had already been established. Mice were evaluated and sacrificed at 4 weeks to asses for fibrosis. (b) Picro-Sirius Red staining of heart coronal sections in mice treated with saline (left), angiotensin II/phenylephrine (center), or angiotensin II/phenylephrine + FAP CAR T cells (right) to evaluate fibrosis (red). Magnification of left ventricular fibrosis (bottom). (c) Quantification of cardiac fibrosis. **** $P<0.0001$ (one-way ANOVA between groups $P<0.0001$; post-hoc multiple comparisons, Tukey's test, $\mathrm{n}=10,9,7$ biologically independent animals, respectively). (d) Comparison of cardiac functional parameters and body weight between experimental and control groups. $* * P<0.01,{ }^{*} P<0.05$, ns $=$ not significant (one-way ANOVA between groups; post-hoc multiple comparisons on significant $(P<0.05)$ ANOVA, Tukey's test, $\mathrm{n}=10,9,7$ biologically independent animals, respectively). Specific $P$ values can be found in the source data. MV E: Mitral valve early 
(E) inflow velocity (e) M mode echocardiography of mice treated with saline (top), angiotensin II/phenylephrine (center), or angiotensin II/phenylephrine + FAP CAR T cells (bottom), (arrows; systole, diastole). Representative images of 2 independent experiments with similar results. All graphs display mean \pm SEM. Scale bars $=100 \mu \mathrm{m}$. 